\title{
Constraining dark sectors at colliders: Beyond the effective theory approach
}

\author{
Philip Harris, ${ }^{1, *}$ Valentin V. Khoze, ${ }^{2, \dagger}$ Michael Spannowsky, ${ }^{2, \sharp}$ and Ciaran Williams ${ }^{3,4,8}$ \\ ${ }^{1}$ CERN, CH-1211 Geneva 23, Switzerland \\ ${ }^{2}$ Institute for Particle Physics Phenomenology, Department of Physics, Durham University, \\ Durham DH1 3LE, United Kingdom \\ ${ }^{3}$ Niels Bohr Institute, University of Copenhagen, Blegdamsvej 17, DK-2100 Copenhagen, Denmark \\ ${ }^{4}$ Department of Physics, University at Buffalo, The State University of New York, \\ Buffalo, New York 14260-1500, USA
}

(Received 25 November 2014; published 10 March 2015)

\begin{abstract}
We outline and investigate a set of benchmark simplified models with the aim of providing a minimal simple framework for an interpretation of the existing and forthcoming searches of dark matter particles at the LHC. The simplified models we consider provide microscopic QFT descriptions of interactions between the Standard Model partons and the dark sector particles mediated by the four basic types of messenger fields: scalar, pseudoscalar, vector and axial-vector. Our benchmark models are characterized by four to five parameters, including the mediator mass and width, the dark matter mass and the effective coupling(s). In the gluon fusion production channel we resolve the top quark in the loop and compute full top-mass effects for scalar and pseudoscalar messengers. We show the LHC limits and reach at 8 and $14 \mathrm{TeV}$ for models with all four messenger types. We also outline the complementarity of direct detection, indirect detection and LHC bounds for dark matter searches. Finally, we investigate the effects which arise from extending the simplified model to include potential new physics contributions in production. Using the scalar mediator as an example, we study the impact of heavy new physics loops which interfere with the top-mediated loops. Our computations are performed within the MCFM framework, and we provide fully flexible public Monte Carlo implementation.
\end{abstract}

DOI: 10.1103/PhysRevD.91.055009

PACS numbers: 95.30.Cq, 12.60.Cn, 12.38.Bx

\section{INTRODUCTION}

Many extensions of the Standard Model (SM) predict the existence of dark particles that can be produced in collisions of ordinary matter but are not directly measurable in the LHC's multipurpose experiments. When measuring the radiation in a scattering event, dark particles would manifest themselves as missing transverse energy, i.e. as an imbalance of the total transverse momentum in the event. Dark particles constitute the dark sector, which plays a special role in any comprehensive beyond-the-StandardModel (BSM) description of particle physics: some of the dark sector particles can be cosmologically stable, ${ }^{1}$ and as such they give rise to the dark matter. Nontrivial dark sectors can also contain massless vector fields which contribute to the dark radiation in the Universe. The fact that dark matter (DM) and dark radiation give the dominant

\footnotetext{
*philip.coleman.harris@cern.ch

valya.khoze@durham.ac.uk

*michael.spannowsky@durham.ac.uk

§ciaran@nbi.dk

${ }^{1}$ This is usually a result of an automatic or imposed discrete symmetry present in the dark sector.
}

Published by the American Physical Society under the terms of the Creative Commons Attribution 3.0 License. Further distribution of this work must maintain attribution to the author(s) and the published article's title, journal citation, and DOI. contributions to the matter and radiation in the Universe is one of the clearest indications that the Standard Model is incomplete and new physics effects must be present in a more fundamental theory of particle interactions.

While dark sectors can be complex, resulting in a rich and varied BSM phenomenology, they have one universal feature which is of particular importance: dark matter particles can interact with visible matter by exchanging a mediator field. When studying scenarios for the production of dark particles at colliders, we consider processes in which a mediator is produced initially in the course of the hadron-hadron collision. This mediator then subsequently decays, either back to SM degrees of freedom or into the dark sector particles. These latter channels will correspond to events with missing transverse energy at colliders. There is no a priori requirement that the mediator decay directly into cosmologically stable DM; all decays into dark particles which are stable at collider scales or do not result in measured displaced vertices will manifest themselves as missing energy. In this sense it is the production and the role of the mediator particle(s) which is of key importance in the collider searches for dark sectors; the actual dark matter is a derivative.

Depending on the nature of the mediator field (arguably the most interesting choices being a vector, axial vector, scalar or a pseudoscalar), different mediator production mechanisms can occur. For vectors and axial vectors, 
acommon assumption is that the dominant production mechanism is the quark-antiquark annihilation at tree level. For scalars and pseudoscalars, on the other hand, the gluon fusion processes are more relevant. This is inspired by the recent Higgs discovery and assumes that the coupling strength of the new scalars to Standard Model fermions is proportional to their SM Yukawa couplings.

Inferring the existence of dark particles in collider experiments requires them to recoil against visible radiation. Since the recoil object need not be essential in the interaction which produces the mediator, a natural candidate for the tagging object is the emission of initial-state radiation, which occurs at a high rate at the LHC. In these monojet signatures a hard jet recoils against the invisible particles. Events with several hard jets are often vetoed, leaving $Z / W+$ jets as major Standard Model backgrounds. In these events the transverse momentum of the jet sets the energy scale of the hard interaction.

The mediating particle can couple directly or indirectly to the initial-state partons; representative diagrams are shown in Fig. 1. The diagram on the far right of Fig. 1 represents an example in which the mediator-SM interaction proceeds via a tree-level interaction with quarks. The mediator can also couple to initial states indirectly; in these instances the underlying production mechanism corresponds to a loop-induced process; the middle diagram in Fig. 1 illustrates this scenario. The propagating loop particle can be integrated out, resulting in an effective dimension-5 operator, illustrated in the leftmost diagram of Fig. 1. This prescription is invalid if $p_{T, j} \gtrsim \mathcal{O}\left(m_{X}\right)$, where $m_{X}$ is the mass of the loop particle. In the case of the top quark, this can readily be achieved. On the other hand, heavy colored states which couple to the mediator can be integrated out provided $\Lambda_{\mathrm{NP}}$ is much larger than the energy scale where the operator is probed.

To be able to probe new physics models with particle masses below the characteristic interaction scale of the hard interaction, so-called simplified models were proposed [1] which only make assumptions on the quantum numbers of particles involved in the minimal processes at the microscopic level, thereby correctly capturing the kinematic features of the new physics model.
The simplified model framework for dark matter and dark sector searches at colliders should constitute a list of key relevant QFT interactions which first produce a mediator particle in a proton-proton collision which subsequently decays into other particles, including dark matter. In general, such benchmark models would be characterized by the production mechanism (e.g. $q \bar{q}$ or gluon-gluon, etc.), the type of the mediator (e.g. scalar, pseudoscalar, vector or axial vector) and the decay channel (e.g. $s$-channel or $t$-channel production of two dark matter fermions, or other DM particle species). Secondly, each individual class of these simple models should be characterized by an appropriately chosen minimal set of physically relevant parameters (coupling constants, masses and widths).

The uses of the simplified model approach in the context of monojet and monophoton searches at colliders and the discussion of its scope have become particularly relevant now in the light of the forthcoming run II of the LHC. The emerging framework is attracting a fair amount of attention in the collider and phenomenology communities. Two recent overviews [2,3] give an example of this. The aim of the present paper is to go beyond the Born-level processes of dark matter production in the quark-antiquark channel and include processes with gluons in the initial state.

The authors of Ref. [2] have discussed examples of tree-level benchmark processes relevant for interpreting DM searches at colliders, specifically quark-antiquark $s$-channel processes mediated by scalar $(\mathrm{S})_{q \bar{q}}^{s}$ and vector $(\mathrm{V})_{q \bar{q}}^{s}$ messengers, and the $t$-channel processes mediated by colored scalar (CS $)_{q \bar{q}}^{t}$ messengers. They have also considered gluon fusion via dimension-5 effective field theory (EFT) operators mediated by scalar $(\mathrm{S})_{g g}^{\mathrm{EFT}}$ and pseudoscalar messengers $(\mathrm{P})_{g g}^{\mathrm{EFT}}$ and have commented on EFT models in which DM coupled preferentially to the third generation.

We will extend these considerations by computing gluon fusion processes at one-loop level in a microscopic theory and apply this analysis to simplified DM models with scalar (S) and pseudoscalar (P) mediators. To enable a direct comparison between models with different mediator types for the LHC reach, we will also reevaluate the predictions of vector $(\mathrm{V})$ and axial-vector (A) mediators produced in the quark-antiquark channel.
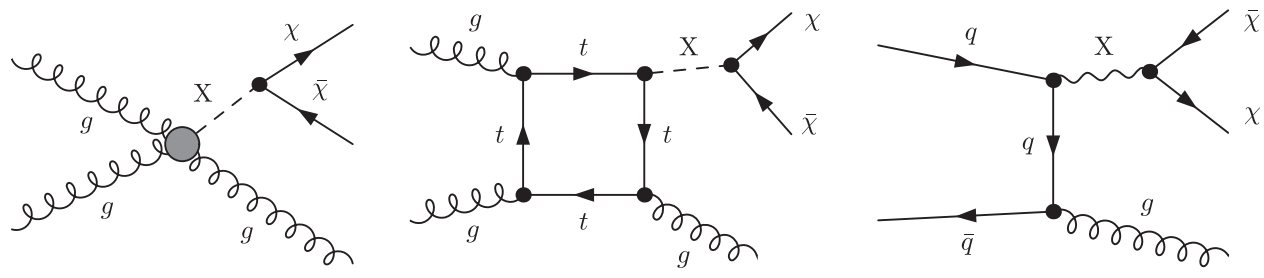

FIG. 1. Representative Feynman graphs for gluon and quark induced monojet processes. The particle $\mathrm{X}$ can be either a scalar, pseudoscalar, vector or axial-vector mediator. The left diagram shows an effective operator approximation of the mediator coupling to gluons. The middle graph represents the full description of the same process, including the fermion mass dependence in the loop, while the right graph depicts a mediator produced in a quark-antiquark annihilation. 
Our work is also complementary to the recent white paper [3] which has concentrated specifically on the cases of vector and axial-vector messengers in the $q \bar{q}$ channel. More generally, i.e. going beyond the point at which specific mediator types are included, Ref. [3] has addressed an important task of identifying the minimal number of relevant parameters characterizing simplified models for DM collider searches. Their proposal is to select four parameters: the DM mass $m_{\mathrm{DM}}$, the mediator mass $m_{\mathrm{MED}}$, and the two coupling constants: $g_{\mathrm{SM}}$, characterizing the coupling of the SM quarks to the mediator, and $g_{\mathrm{DM}}$, which is the coupling of the mediator to dark particles.

We would like to emphasize here the importance of the mediator width $\Gamma_{\mathrm{MED}}$, which we will treat as an independent parameter inherent in the characterization of the simplified models. The impact of $\Gamma_{\mathrm{MED}}$ in monojet searches has already been discussed in earlier literature, see e.g. Refs. [4-8]. The DM production cross section at colliders scales numerically as $\sigma \propto g_{\mathrm{SM}}^{2} g_{\mathrm{DM}}^{2} /\left(m_{\mathrm{MED}}^{4} \Gamma_{\mathrm{MED}}\right)$, i.e. inversely proportional to the width, leading to a resonant enhancement of the cross section at small values of $\Gamma_{\mathrm{MED}}$, as pointed out in Ref. [3].

In the approach of Refs. [3,9], the messenger width was computed within the simplified model itself. But this assumes that the messenger can only decay into the DM particles as well as the $q \bar{q}$ pairs from which it was produced in the first place. This is a strong assumption which we are not prepared to apply universally, as this would exclude the possibility of mediators decaying into anything except a single species of the cosmologically stable DM within the dark sector (and would also limit the decay possibilities into SM particles). Instead, as already pointed out above, we will treat $\Gamma_{\text {MED }}$ as a free parameter which we will vary and whose minimal value should not be less than the calculated width into DM and the appropriate SM channels. ${ }^{2}$ We advocate the approach with four parameters: $m_{\mathrm{MED}}, \Gamma_{\mathrm{MED}}, m_{\mathrm{DM}}$ and the product of the couplings of the mediator to the SM and to DM particles, $g_{\mathrm{eff}}^{2}$. In order to provide a fully flexible Monte Carlo tool for experimental studies we have implemented the models described above in the MCFM framework [13-15]. The results of this paper extend the existing dark matter processes in MCFM [6], which focused primarily on NLO corrections in the effective field theory approach (matching to parton showers was achieved in Ref. [16]). The results we present here will be available in the next public release of MCFM.

This paper is organized as follows: We first briefly discuss the limitations of the EFT approach at collider

\footnotetext{
${ }^{2}$ Recently, while this paper was being finalized, Ref. [10] appeared, considering scalar and pseudoscalar mediators in the gluon fusion channel and also pointing out the importance of keeping the mediator width a free parameter. Limits for invisible decays of the Higgs boson in monojet measurements, taking the full top-mass dependence into account, have been obtained in recent years $[11,12]$.
}

searches and proceed to define and set up the simplified models we study in Sec. II. In Sec. III we assemble the necessary formulas for DM scattering cross sections for our models relevant to direct detection and indirect detection of DM experiments. In Sec. IV we discuss the event generation and reconstruction as well as existing measurements for monojet final states, and we proceed to present limits and projections for our simplified models. Following this, in Sec. V we extend our simplified models to allow for the possibility of additional contributions of new very heavy particles to the mediator production mechanism from initial-state gluons. In the Appendix, for the convenience of the reader, we list the basic amplitudes for a scalar mediator plus jet production in the spinor helicity formalism. Our conclusions are presented in Sec. VI.

\section{SIMPLIFIED MODELS}

In this article we focus primarily on monojet searches, induced by a mediator interaction between gluons and dark particles. We stress here that collider limits apply whether the dark particles are dark matter candidates or not, i.e. no assumption on their cosmic abundance or astrophysical production mechanism is required.

The promising kinematics of the monojet signature [17-21] was appreciated for new physics searches several years ago [22-25]. Models studied include those with extra dimensions [26] to compressed SUSY spectra [27-29]. More recently it was also argued that this configuration can constrain dark sectors in a fairly model-independent way $[5,25,30-34]$. To limit the number of free parameters and to facilitate the interpretation and cross correlation of measurements at colliders and direct detection experiments, effective operators were proposed to parametrize the signal hypotheses, i.e. the contributions of new physics models.

A priori, following the discussion in Ref. [35], the use of effective operators in constraining new physics scenarios in a fairly model-independent way is a legitimate approach in parts of the model parameter space. Differential distributions can always be used to constrain the Wilson coefficients $C_{i}$ of specific effective operators $O_{i}$. It needs to be kept in mind, however, that these constraints are only meaningful when the scale at which the operators are probed is below the validity region of the effective theory, e.g. $\sqrt{\hat{s}} \ll \Lambda_{\mathrm{NP}}$. This constraint results in the red vertical line of Fig. 2. Because $C_{i} \sim g_{\mathrm{NP}} / \Lambda_{\mathrm{NP}}$, a constraint from a measurement on the Wilson coefficient translates into a diagonal curve, depicted in black in Fig. 2, resulting in four regions of the parameter space of new physics models. While the sectors left of the vertical red line are outside the validity range of the effective theory, only the models that fall into the green region could be constrained by the measurement. More specifically, when aiming for an interpretation of the constraint on the effective operator in terms of a new physics model, models that are constrained have to have a high new physics scale, i.e. 


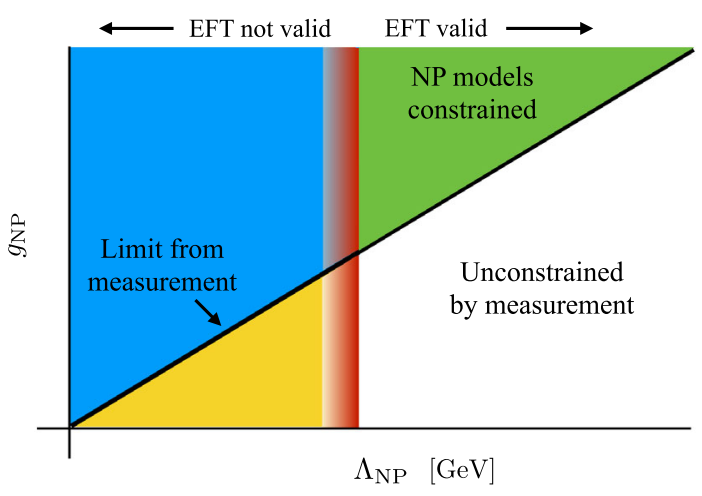

FIG. 2 (color online). Figure taken from Ref. [35], schematically illustrating the valid interpretations of experimental results in terms of EFTs. Experimental analyses set a bound on the $g_{\mathrm{NP}} / \Lambda$, corresponding to the solid line; combinations of $g_{\mathrm{NP}}$ and $\Lambda$ greater than this bound are excluded. However, if the experiment was able to probe the scale of the new physics, then the EFT prescription was invalid; this corresponds to all values left of the vertical line.

$\sqrt{\hat{s}}<\Lambda_{\mathrm{NP}} \simeq m_{\mathrm{NP}}$, and a large coupling $g_{\mathrm{NP}}$. This can require the dark particles to be strongly coupled to the visible sector, which further complicates the interpretation.

Therefore, a reliable interpretation of collider searches of dark matter particles should be based on basic QFT interactions where all intermediate propagating degrees of freedom in a given process are explicitly taken into account [2,36-39]. Unsurprisingly, contributions due to light degrees of freedom have been found to be significant for limit setting [4,6,8,40-42].

In order to model mediator production, we will consider simplified models with the mediators to the dark sector associated with scalar $S$, pseudoscalar $P$, vector $Z^{\prime}$ and axial-vector $Z^{\prime \prime}$ fields with interactions:

$$
\begin{aligned}
\mathcal{L}_{\text {scalar }} \supset-\frac{1}{2} m_{\mathrm{MED}}^{2} S^{2}- & g_{\mathrm{DM}} S \bar{\chi} \chi-g_{\mathrm{SM}}^{t} S \bar{t} t-g_{\mathrm{SM}}^{b} S \bar{b} b, \\
\mathcal{L}_{\text {pseudo-scalar }} \supset & -\frac{1}{2} m_{\mathrm{MED}}^{2} P^{2}-g_{\mathrm{DM}} P \bar{\chi} \gamma^{5} \chi \\
& -g_{\mathrm{SM}}^{t} P \bar{t} \gamma^{5} t-g_{\mathrm{SM}}^{b} P \bar{b} \gamma^{5} b
\end{aligned}
$$

$\mathcal{L}_{\text {vector }} \supset \frac{1}{2} m_{\mathrm{MED}}^{2} Z_{\mu}^{\prime} Z^{\prime \mu}-g_{\mathrm{DM}} Z_{\mu}^{\prime} \bar{\chi} \gamma^{\mu} \chi-\sum_{q} g_{\mathrm{SM}}^{q} Z_{\mu}^{\prime} \bar{q} \gamma^{\mu} q$,

$\mathcal{L}_{\text {axial }} \supset \frac{1}{2} m_{\mathrm{MED}}^{2} Z_{\mu}^{\prime \prime} Z^{\prime \mu}-g_{\mathrm{DM}} Z_{\mu}^{\prime \prime} \bar{\chi} \gamma^{\mu} \gamma^{5} \chi-\sum_{q} g_{\mathrm{SM}}^{q} Z_{\mu}^{\prime \prime} \bar{q} \gamma^{\mu} \gamma^{5} q$

Two types of coupling constants appear in these equations: $g_{\mathrm{SM}}$, which collectively denote the couplings between messenger fields and Standard Model particles, and $g_{\mathrm{DM}}$, which are couplings of the messenger to the dark sector $\chi$ particles. We have assumed that the scalar and pseudoscalar messengers are coupled only to top and bottom quarks with the Yukawa-type coupling denoted $g_{\mathrm{SM}}^{t, b}$ in Eqs. (1) and (2) - these are the dominant interactions of (pseudo) scalars with the SM fermions; in fact, in most cases only the couplings to tops are important. Phenomenologically this resembles models with minimal flavor violation [43] and a SM-Yukawa-like hierarchy for the mediator-fermion interactions. The couplings of messengers to all six flavors of SM quarks are taken to be proportional to the corresponding Higgs Yukawa couplings, $y_{q}$, and to make our definitions look symmetric we choose to parametrize the DM couplings in a similar fashion, so that

for scalar \& pseudoscalar messengers: $g_{\mathrm{SM}}^{q} \equiv g_{q} y_{q}$,

$g_{\mathrm{DM}} \equiv g_{\chi} y_{\chi}, \quad$ where $y_{\chi} \equiv \frac{m_{\chi}}{v}=\frac{m_{\mathrm{DM}}}{v}$.

The product of the top and $\chi$ couplings to messengers is

$$
g_{\mathrm{eff}}^{2}:=g_{\mathrm{SM}}^{q} g_{\mathrm{DM}}=g_{t} g_{\chi} y_{t} y_{\chi}=g_{q} g_{\chi} \frac{m_{t} m_{\mathrm{DM}}}{v^{2}},
$$

and we keep the scaling $g_{q}$ flavor universal for all quarks, so $g_{t}=g_{q}$.

All vectors and axial vectors are assumed to be coupled to all quarks uniformly, hence the sums in Eqs. (3) and (4) are over all quark flavors (with a universal gauge-type coupling denoted $\left.g_{\mathrm{SM}}\right)$. For the axial-vector and vector mediators we will use

$$
g_{\mathrm{eff}}^{2}:=g_{\mathrm{SM}} g_{\mathrm{DM}} .
$$

In our setup, the Standard Model particles only interact via the mediator with the invisible sector, i.e. the particle $\chi$. Thus, all amplitudes contributing to the processes we will study in Secs. III-V are proportional to $g_{\text {eff }}^{2}$ defined in Eqs. (6) and (7).

It is important to stress that models derived from scalar and pseudoscalar mediators provide some of the simplest realizations of a nonminimal Higgs sector in which the Standard Model Higgs interacts and can mix with the scalar mediators. Following the Higgs discovery, there is a renewed interest in the literature in Higgs portal models where the scalar mediators are SM singlets but the SM Higgs $h$ interacts with them via the interaction $\lambda_{\mathrm{hp}}|H|^{2}|\Phi|^{2}$. The Higgs portal models with singlet scalar messengers will be treated in the same way as general scalar messengers. These models provide a direct link with Higgs physics and also include theoretical scenarios which manifest a common origin of the electroweak and the DM scales in nature as was recently explored in Refs. [44-53]. 
More specifically, consider the case where the mediator is a complex scalar $\Phi$ which is a singlet of the SM and interacts with it only via the portal interactions with the Higgs:

$$
\mathcal{L}_{\text {portal }}=\lambda_{\text {hp }}|H|^{2}|\Phi|^{2}-g_{\mathrm{DM}} \bar{\chi} \Phi \chi
$$

Furthermore, we assume that $\Phi$ is charged under the gauge group of the dark sector and is coupled to other dark particles [which in (8) for simplicity are taken to be the dark fermions $\chi$ and $\bar{\chi}$, but this can be extended to include vector and scalar dark particles]. In models which contain no input mass scales in the microscopic Lagrangian, the vacuum expectation value for the field $\Phi$ can be generated quantum mechanically, e.g. via the Coleman-Weinberg mechanism [54] in the dark sector as explained in Ref. [44]. The VEV $\langle\Phi\rangle$ then induces the vacuum expectation value $v$ for the Higgs field via the portal interaction in Eq. (8) and triggers electroweak symmetry breaking. It also generates the mass scale $m_{\mathrm{DM}}=g_{\mathrm{DM}}\langle\Phi\rangle$ in the dark sector. Thus, in this class of models the electroweak scale and dark matter scale have a common origin. To see that such Higgs portal models continue to be described effectively by the minimal simplified model in Eq. (1), we rewrite (8) after electroweak symmetry breaking, in unitary gauge, as

$$
\begin{aligned}
\mathcal{L}_{\text {portal }} \supset & 2 \lambda_{\mathrm{hp}}\langle\Phi\rangle v \phi h+\lambda_{\mathrm{hp}} v^{2} \phi^{2}+\lambda_{\mathrm{hp}}\langle\Phi\rangle^{2} h^{2} \\
& -g_{\mathrm{DM}} \bar{\chi}(\langle\Phi\rangle+\phi) \chi .
\end{aligned}
$$

Transforming into the mass eigenstate basis, we find two scalar resonances $h_{1}$ and $h_{2}$, both of which interact with the Standard Model and the dark sector. Either state can be identified with $S$ in Eq. (1). ${ }^{3}$

Following a similar line of reasoning for the case where the pseudoscalar can develop a VEV and CP is not a good quantum number, we can map the Higgs portal interactions to the form of Eq. (2).

We note that the scalar and pseudoscalar Lagrangians defined above are compatible with the principle of minimal flavor violation. If, on the other hand, one were to relax this constraint, one could define a scalar (and pseudoscalar) Lagrangian in which the mediating particle couples directly to the light quark species, with no Yukawa suppression. In these instances the phenomenology of the signal changes substantially, since the production mechanism is now identical to vector and axial-vector mediators. As a result, the phenomenology of these signatures (LHC limits and cross sections) are similar in size to those obtained using the vector and axial mediators (the major differences arising

\footnotetext{
${ }^{3}$ For simplicity and concreteness, this paper concentrates on the simplified models with mediators coupled to Dirac fermions $\chi$ in the dark sector. These models can be extended to incorporate scalar and vector dark matter particles as in Refs. [46-48] and chiral fermions.
}

from a scalar mediator in an isotropic final state with no spin correlations). We note that these processes are available in MCFM [6], and the analysis we present here could be applied easily to these models; however, for brevity we do not consider them in this paper.

\section{A. The mediator width}

We would now like to discuss the impact of the mediator width in our simplified models. Given the models specified in Eqs. (1)-(4) with democratic quark-(axial-)vector and Yukawa-type quark-scalar interactions, we obtain a lower limit for the width of the mediator. For scalar and pseudoscalar mediators, depending on their mass, decays to heavy quarks may or may not be open (i.e. $m_{\mathrm{MED}}$ is required to be $>2 m_{t}$ for an open decay). In certain regions of parameter space, loop-induced decays to vector bosons, or extended dark sector decays, and off-shell decays (e.g. to $t^{*} \bar{t}$ ), may significantly enhance the "minimal widths" which we define as

$$
\begin{gathered}
\Gamma_{\mathrm{MED}, \min }^{V, A}=\Gamma_{\chi \bar{\chi}}^{V, A}+\sum_{i=1}^{N_{f}} N_{c} \Gamma_{q_{i}}^{V, A} \bar{q}_{i}+N_{c} \Gamma_{t \bar{t}}^{V, A}, \\
\Gamma_{\mathrm{MED}, \min }^{S, P}=\Gamma_{\chi \bar{\chi}}^{S, P}+N_{c} \Gamma_{t \bar{t}}^{S, P},
\end{gathered}
$$

where $\Gamma_{\chi \bar{\chi}}$ is the mediator decay rate into two DM particles (which here we assume are fermions $\chi \bar{\chi}$; modifications to scalar dark matter are trivial to incorporate). The sum on the right-hand side of the first equation is over the massless SM quark flavors interacting with the vector and axial-vector mediators. These widths are lower bounds on the total, and as such we treat the width as a free parameter and investigate the LHC phenomenology as a function of the rescaled width. For decays into fermions, the partial widths are defined as follows:

$$
\begin{gathered}
\Gamma_{f \bar{f}}^{V}=\frac{g_{f}^{2}\left(m_{\mathrm{MED}}^{2}+2 m_{f}^{2}\right)}{12 \pi m_{\mathrm{MED}}} \sqrt{1-\frac{4 m_{f}^{2}}{m_{\mathrm{MED}}^{2}}}, \\
\Gamma_{f \bar{f}}^{A}=\frac{g_{f}^{2}\left(m_{\mathrm{MED}}^{2}-4 m_{f}^{2}\right)}{12 \pi m_{\mathrm{MED}}} \sqrt{1-\frac{4 m_{f}^{2}}{m_{\mathrm{MED}}^{2}}}, \\
\Gamma_{f \bar{f}}^{S}=\frac{g_{f}^{2} m_{f}^{2} m_{\mathrm{MED}}}{8 \pi v^{2}}\left(1-\frac{4 m_{f}^{2}}{m_{\mathrm{MED}}^{2}}\right)^{\frac{3}{2}}, \\
\Gamma_{f \bar{f}}^{P}=\frac{g_{f}^{2} m_{f}^{2} m_{\mathrm{MED}}}{8 \pi v^{2}}\left(1-\frac{4 m_{f}^{2}}{m_{\mathrm{MED}}^{2}}\right)^{\frac{1}{2}},
\end{gathered}
$$

where $m_{f}$ denotes masses of either SM quarks $q$ or DM fermions $\chi$, and the coupling constant $g_{f}$ denotes either $g_{q}$ or $g_{\chi}$, as defined on the right-hand side of Eq. (5). In Fig. 3 we plot the minimal widths computed using Eqs. (10)-(15) 

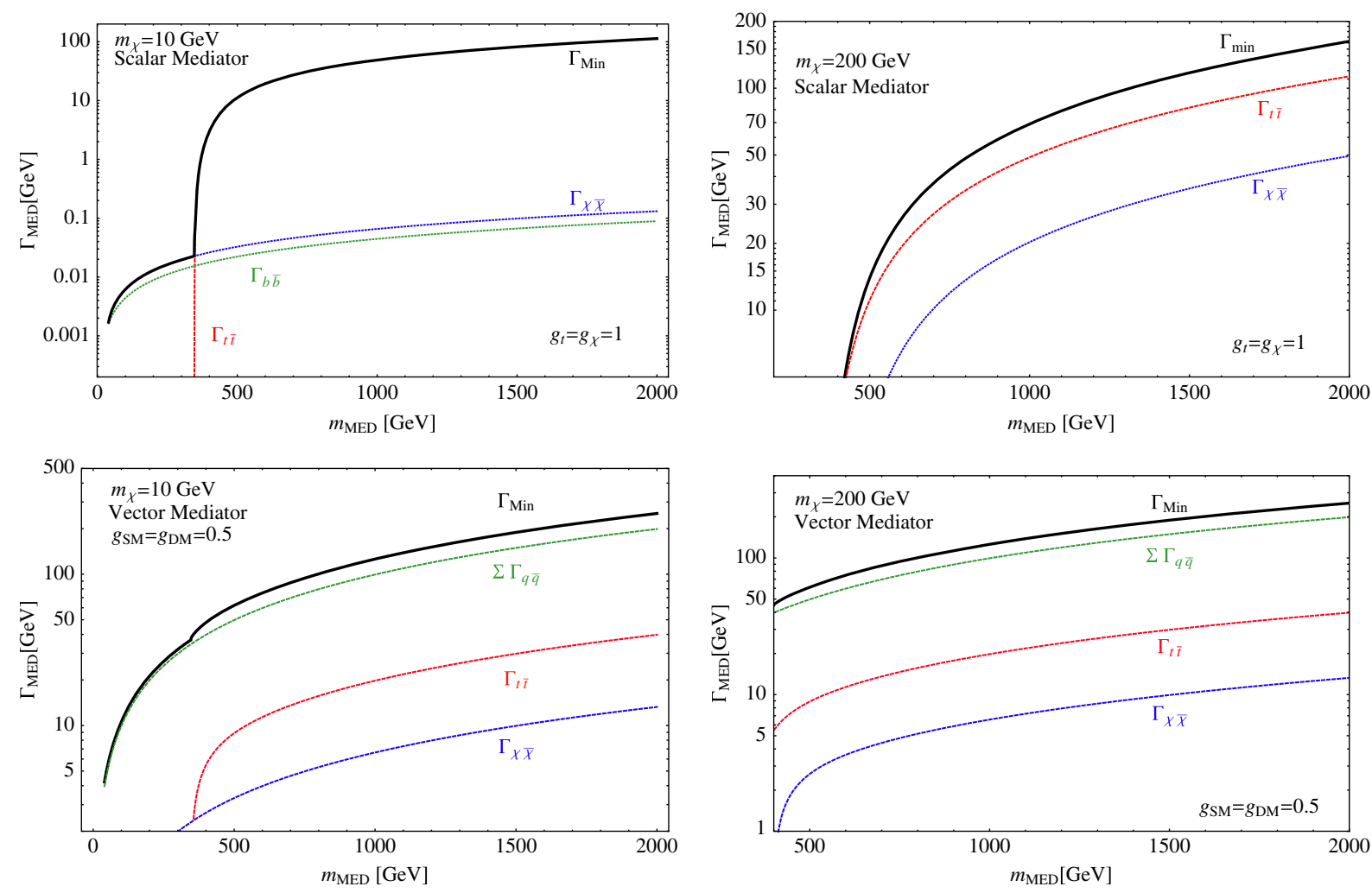

FIG. 3 (color online). Minimal width as a function of the mediator mass for scalar and vector operators, with two different DM mass choices. Individual partial width contributions are illustrated.

for scalar and vector types of the mediators as functions of the mediator mass, for two representative choices of DM masses. As expected, the (pseudo)scalar models parametrized in terms of Yukawa couplings are much more sensitive to the choice of DM mass. The hadronic branching ratio for the vector mediator dominates the decays (due to the combination of light flavors and color factors $N_{f} N_{c}$ ), extended dark sectors could result in larger branching ratios to the dark sector and thus increase the width. For the scalar there are no light decays (apart from $b \bar{b}$, which can become important for light mediators), and the relative enhancement/suppression of $t \bar{t}$ decays scales like $N_{c}\left(m_{t} / m_{\mathrm{DM}}\right)^{2}$.

In summary: The set of simplified models for dark particle searches we study is defined by Eqs. (1)-(4). It automatically accounts for Higgs portal models with scalar and pseudoscalar messengers. In Sec. V we will further extend the model in Eq. (1) by adding a new BSM interaction (21).

Our simplified models are characterized by the type of the mediator field, which can be a scalar, pseudoscalar, vector or axial-vector particle. There are four (five) types of input parameters involved in this description: the mediator mass $m_{\mathrm{DM}}$, the mediator width $\Gamma_{\mathrm{DM}}$, the dark particle mass $m_{\mathrm{DM}}$ and an appropriately defined coupling constant (or constants) to characterize the combined effect of the SMmediator and the mediator-dark-sector interaction.
We use $g_{\mathrm{SM}} g_{\mathrm{DM}}$ as the input effective coupling parameter for the vector and axial-vector cases (3)-(4). In the cases of scalar and pseudoscalar mediators (1)-(2) we choose to scale the couplings with the SM Yukawas and use the product of scaling factors $g_{q} g_{\chi}$ defined in (5) as the input effective coupling parameter. ${ }^{4}$ (The extended model studied in Sec. V will contain an additional coupling $g_{g}$.)

\section{DIRECT AND INDIRECT DETECTION LIMITS}

If we make the assumption that the particle $\chi$ of Eqs. (1)-(4) is a dark matter candidate, accounting for the observed dark matter abundance in the Universe, we can derive limits on our simplified models from low-energy interactions, i.e. direct and indirect detection experiments. Direct detection experiments measure the recoil of the nucleus of which the dark matter particle scatters off. Our limits are based on measurements by LUX [55-57], which

\footnotetext{
${ }^{4}$ The choice of what is treated as the input coupling parameter for (pseudo)scalar mediators, namely the combination $\left(g_{\mathrm{SM}} / y_{q}\right)$ $\left(g_{\mathrm{DM}} / y_{\chi}\right)$, or $\left(g_{\mathrm{SM}} / y_{q}\right) g_{\mathrm{DM}}$, or the original couplings $g_{\mathrm{SM}} g_{\mathrm{DM}}$, is of course only a simple reparametrization which only affects which dimensionless parameters are held fixed when one varies the mass parameters for the mediators/dark particles in the plots. We have chosen the first combination, and the authors of Ref. [10] used the second, while the vector cases of course have no Yukawas to scale. In any case, this is a simple reparametrization.
} 
currently provides the strongest bounds for $m_{\mathrm{DM}} \gtrsim 6 \mathrm{GeV}$. In these settings dark matter particles are assumed to be nonrelativistic, the momentum transfer (depicted in the right diagram of Fig. 1) is small and describing the interaction in terms of effective operators is justified as long as $\mathcal{O}\left(m_{\mathrm{MED}}\right) \gtrsim 1 \mathrm{GeV}$.

For the calculation of the scattering cross section of a dark matter particle scattering spin independently via a vector mediator off a proton, we find

$$
\sigma_{\chi \mathrm{p}}^{V}=\frac{9}{\pi} \frac{g_{\mathrm{DM}}^{2} g_{\mathrm{SM}}^{2} \rho^{2}}{m_{\mathrm{MED}}^{4}}
$$

and for the scalar, interacting with the nuclei only via the gluons, we use [58-60]

$$
\sigma_{\chi \mathrm{p}}^{S}=\frac{\rho^{2}}{\pi}\left|\frac{m_{p}}{m_{t}} \frac{g_{t} y_{t} g_{\chi} y_{\chi}}{m_{\mathrm{MED}}^{2}} \frac{2}{27} f_{\mathrm{TG}}\right|^{2}
$$

where $\rho=m_{\mathrm{DM}} m_{p} /\left(m_{\mathrm{DM}}+m_{p}\right)$ is the reduced mass and $f_{\mathrm{TG}} \simeq 0.9[61]$.

Axial-vector mediators result in spin-dependent darkmatter-proton scatterings, with the cross section described by [62]

$$
\sigma_{\chi \mathrm{p}}^{A}=\frac{3}{\pi} \frac{g_{\mathrm{DM}}^{2} g_{\mathrm{SM}}^{2} a^{2} \rho^{2}}{m_{\mathrm{MED}}^{4}}
$$

with $a=\Delta u+\Delta d+\Delta s \simeq 0.43[8,61]$, assuming democratic couplings to all quark flavors. We compare the predicted cross sections with the combined bounds of WIMP-proton-scattering limits of PICASSO [63], COUPP [64] and SIMPLE [65].

While direct detection experiments can give strong constraints for the vectors and the scalar mediator, the scattering of a pseudoscalar off a nucleus is strongly velocity dependent and vanishes in the nonrelativistic limit. Therefore, for pseudoscalars, taking existing limits into account $[66,67]$, indirect detection experiments can result in stronger bounds than direct detection experiments $[68,69]$. For the simplified model of Eq. (2), using the s-wave velocity-averaged DM annihilation cross section into $\bar{b} b$,

$$
\langle\sigma v\rangle_{\bar{b} b}^{P}=\frac{N_{C}}{2 \pi} \frac{\left(y_{b} g_{b}\right)^{2}\left(y_{\chi} g_{\chi}\right)^{2} m_{\mathrm{DM}}^{2}}{\left(m_{\mathrm{MED}}^{2}-4 m_{\mathrm{DM}}^{2}\right)^{2}+m_{\mathrm{MED}}^{2} \Gamma_{\mathrm{MED}}^{2}} \sqrt{1-\frac{m_{b}^{2}}{m_{\mathrm{DM}}^{2}}},
$$

we can derive a bound on the parameters in the $\bar{b} b$ channel [66]. As always, the scaling factors $g_{q}$ are kept flavor universal, so $g_{b}=g_{t}=g_{q}$ in these equations.

In all four expressions for the cross sections (16)-(19) for scalar and pseudoscalar mediators we will keep $g_{q}$ and $g_{\chi}$ fixed, while for vector and axial-vector mediators we fix $g_{\mathrm{SM}}$ and $g_{\mathrm{DM}}$.

\section{SEARCHES AT THE LHC}

\section{A. Event generation and final-state reconstruction}

We generate the processes $p p \rightarrow(X \rightarrow \bar{\chi} \chi)+$ jet where $X$ can be a vector, axial vector, scalar or pseudoscalar using MCFM. The existing implementation of DM process in MCFM (described in Ref. [6]) consisted of NLO predictions for vector, axial-vector, scalar and pseudoscalar mediators in the effective field theory prescription; in addition, LO processes involving a scalar mediator and top quark loop were also included. In the previous version, propagating resonances were included for the above process in an ad hoc manner in which the user specified the value of the width, mediator mass and couplings, in addition to the mass of the dark matter particle. We have extended the code to include the LO process in which a pseudoscalar mediator couples to the top quark loop, and parameterized the code in terms of the simplified models described in the previous section. In addition, we have included heavy new physics in the loop for scalar and pseudoscalar mediators, but we postpone discussion of these effects to the next section. These extensions to the code will be released publicly in the next version of MCFM.

The generated signal samples are showered using Pythia 8 [70] with tune 4C. The background yield for our $8 \mathrm{TeV}$ limits is entirely inferred from the CMS monojet searches [20]. To emulate the detector performance, the showered samples are clustered using the anti- $k_{T}$ [71] algorithm with a cone size of 0.4 . The resulting three leading jets with a $p_{T}$ above $15 \mathrm{GeV}$ are smeared following the resolution functions quoted by CMS [72]. The remaining hadronic recoil is smeared following the MET resolution quoted in Ref. [73].

For the $14 \mathrm{TeV}$ analysis, the two leading backgrounds $(Z \rightarrow \nu \nu$ and $W \rightarrow \ell \nu)$ are generated using MadGraph [74] and are smeared according to the same scheme discussed in the previous paragraph. From these samples a kinematic scale factor is obtained. The cross sections for all other process, excluding the $t \bar{b}$ and single top, are obtained by scaling the $8 \mathrm{TeV}$ predictions in the CMS analysis by the NLO scale factor from $8 \mathrm{TeV}$ to $14 \mathrm{TeV}$ obtained in MCFM [14]. For the top and $t \bar{t}$ backgrounds, the partial NNLO cross sections are used [75].

To be able to reinterpret the CMS monojet search in terms of the simplified models of Eqs. (1)-(4), we follow the event selection of Ref. [20] closely. The signal extraction region requires a $E_{\mathrm{T}}^{\text {miss }}>200 \mathrm{GeV}$, which is beyond the plateau efficiency and thus very close to $100 \%$. The hardest jet in the event must fulfill $p_{T, j_{1}} \geq 110 \mathrm{GeV}$ and $|\eta|<2.4$. We accept events with a second jet if $p_{T, j_{2}} \geq 30 \mathrm{GeV},|\eta|<5.0$ and provided the azimuthal 

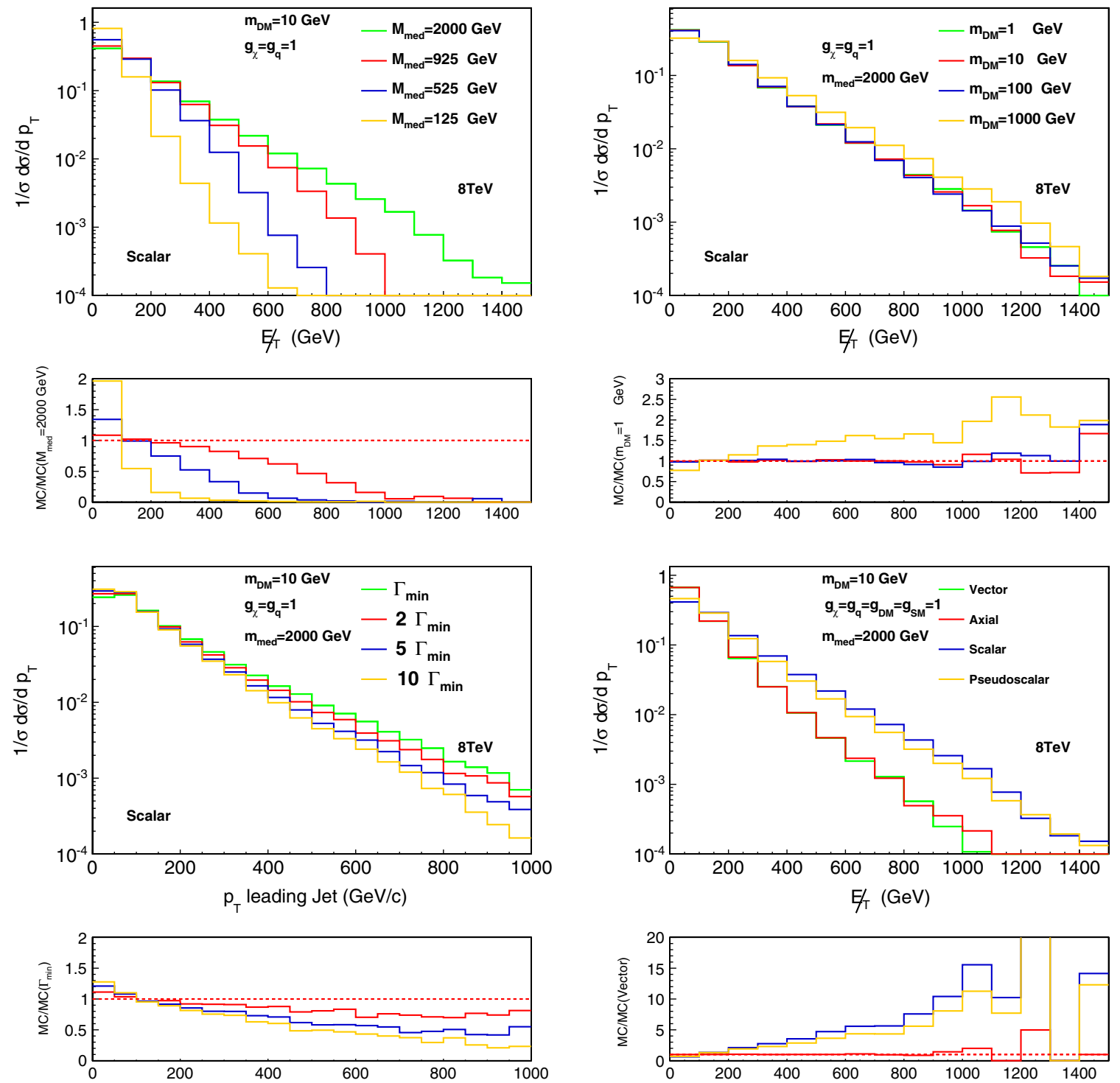

FIG. 4 (color online). Kinematics and reconstruction of selected distributions. Events are generated using MCFM and PYTHIA; detector level effects are also simulated. Plots are normalized to have the same yield so that kinematic differences can be observed. We set the coupling parameters defined in Eq. (5) to $g_{\chi}=1=g_{q}$ for (pseudo)scalars and $g_{\mathrm{DM}}=1=g_{\mathrm{SM}}$ for (axial-)vector mediators.

angle between the two jets is less than 1.8 radians, i.e. $\Delta \phi_{j_{1}, j_{2}}<1.8$. We veto events with more than two jets. To optimize the sensitivity of the analysis with respect to varying dark matter and mediator masses, the exclusion limits are based on seven phase space regions distinguished by $E_{\mathrm{T}}^{\text {miss }}>(250,300,350,400,450,500,550) \mathrm{GeV}$.

The limits shown in this section are based on the most sensitive of the seven regions, respectively. For each signal model, the seven regions are scanned, taking the most sensitive result obtained by scanning the individual regions. The limit computation is preformed in the same manner as that in the CMS analysis, profiling the likelihood and obtaining the $90 \%$ confidence level using the $\mathrm{CL}_{S}$ procedure. Both the systematics and statistical uncertainties as described in the CMS monojet analysis are taken into account. As a cross check, the cross section limit results were reproduced to within $3 \%$. For $14 \mathrm{TeV}$ we use the same reconstruction approach, keeping the same systematics, but scaling the yields by the predicted scale factors.

In Fig. 4 we present a series of differential distributions obtained using the prescription defined above, focusing primarily on the case of a scalar mediator. Most of the kinematic properties can be inferred from the on-shell condition for the mediator, namely

$$
\left(E_{T}^{\min }\right)^{2}+4 m_{\mathrm{DM}}^{2}<s_{\chi \bar{\chi}} \sim M_{X}^{2}
$$

As the mediator particle becomes heavier, the missing transverse energy spectrum becomes harder, as illustrated by the top-left plot in Fig. 4. The lower-left plot in Fig. 4 illustrates the jet $p_{T}$ dependence on the width. Broader widths result in a spreading of $s_{\chi \bar{\chi}}$; however, events which 
become more energetic are additionally damped by PDF suppression. Therefore, as particles acquire broader widths, distributions are naturally softened relative to the narrowwidth case. Finally, the lower-right plot in Fig. 4 illustrates the differences in the MET spectrum associated with the production mechanism; the gluon induced scalar and pseudoscalar processes result in a harder spectrum.

Small kinematic differences are observed between each of the gluon induced processes or vector induced processes. The largest modifications to the kinematic shape come from variations in the mediator mass, and variation in the width. When scanning the dark matter mass, visible modifications of the kinematics shapes are only present for off-shell masses.

\section{B. Experimental searches}

In Fig. 5 we plot the limits on the LHC cross section at $8 \mathrm{TeV}$ and projected limits at $14 \mathrm{TeV}$ for scalar,
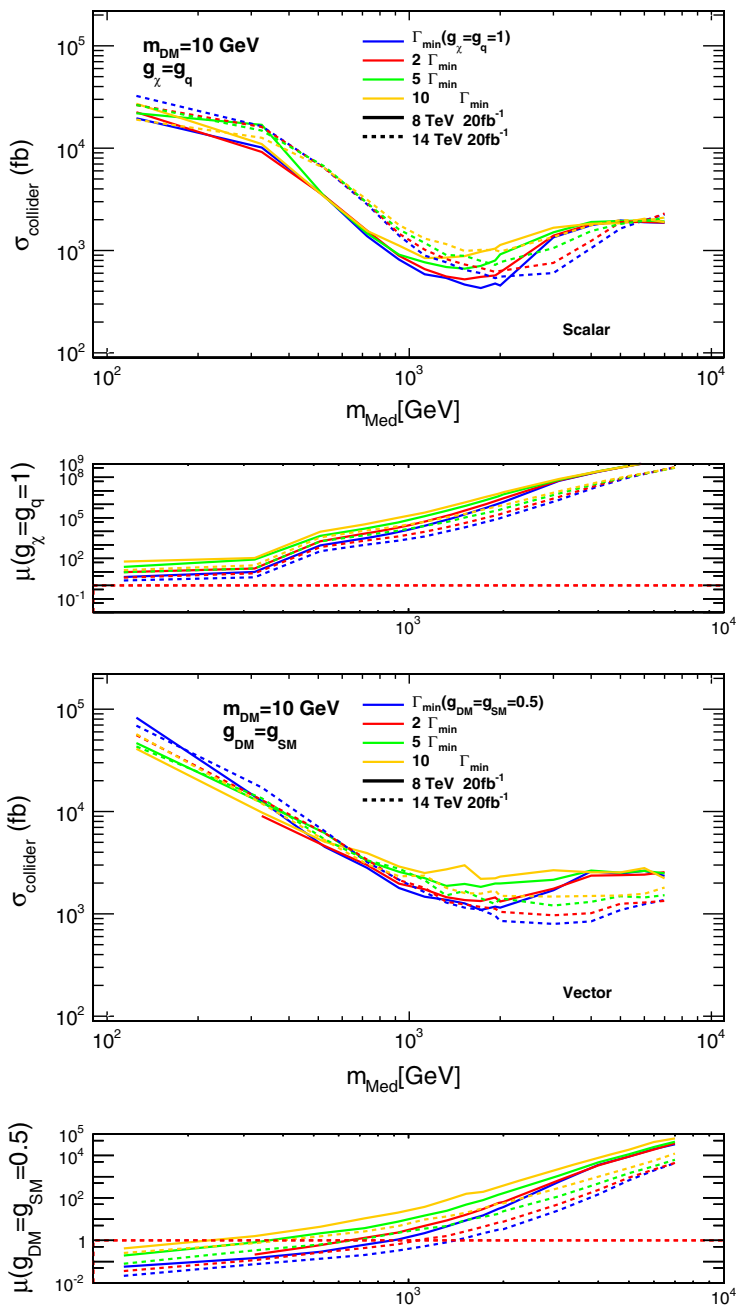

pseudoscalar, vector and axial-vector mediators, as the function of the mediator mass. For the $\chi$-particle dark matter mass we have chosen a relatively small value of $10 \mathrm{GeV}$, although the results obtained for heavier dark matter were found to be similar. The kinematics of the process is then completely specified once a coupling is set, since this fixes the minimal width of the mediator. For the coupling parameters we choose $g_{g}=g_{\chi}=1$ in the scalar and pseudoscalar case, and $g_{\mathrm{SM}}=g_{\mathrm{DM}}=0.5$ for (axial) vectors. With the kinematics of the model fixed, the properties of the model allow a limit on a cross section to be derived. From the derived limit, a value, $\mu$, is obtained which refers to the ratio of the excluded cross section with respect to the predicted cross section as determined by the couplings and width constraint. Translating the constraint on $\mu$ to direct constraints on the couplings, $g$, requires propagating the cross section dependence of both the couplings and the width. Values with $\mu<1$ typically
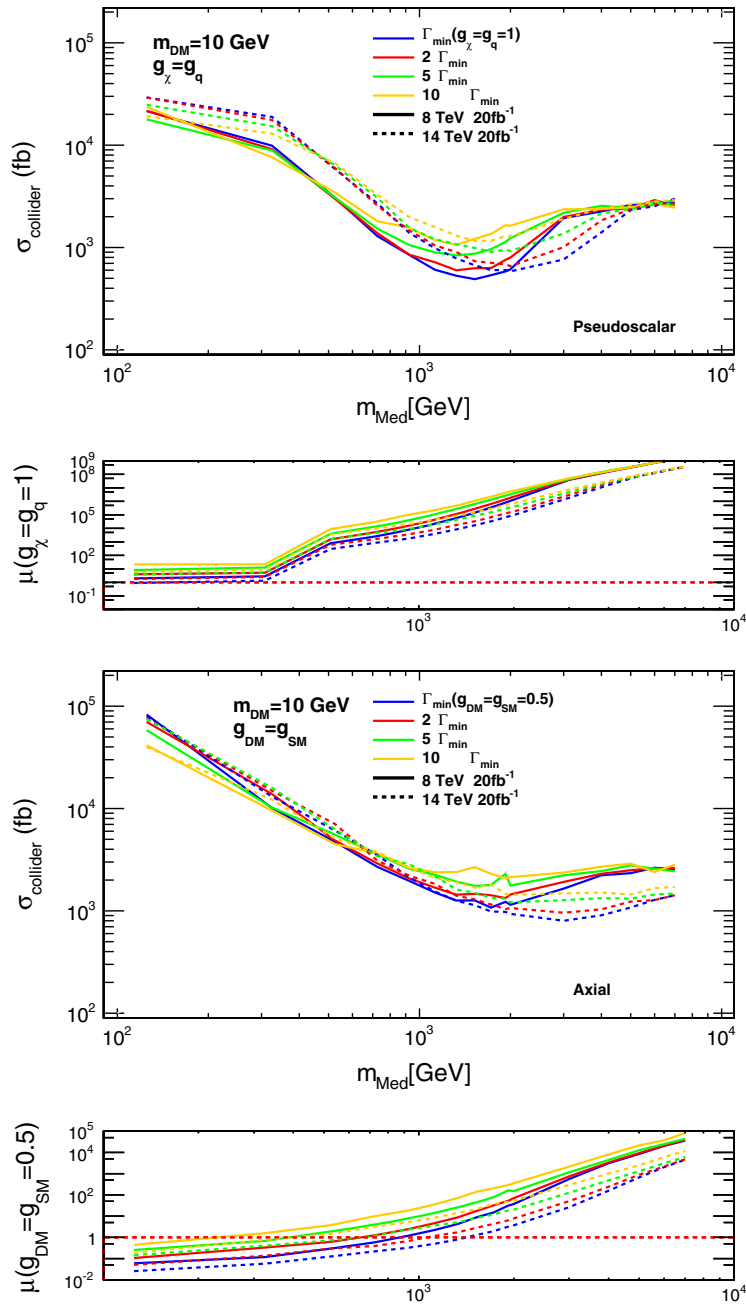

FIG. 5 (color online). Cross section limit bounds (projections) for LHC cross sections at $8 \mathrm{TeV}$ (14 TeV) for scalar (upper left), pseudoscalar (upper right), vector (lower left) and axial vector (lower right). We chose $m_{\mathrm{DM}}=10 \mathrm{GeV}$ and set the coupling parameters defined in Eq. (5) to $g_{\chi}=1=g_{q}$ for (pseudo)scalars and $g_{\mathrm{DM}}=0.5=g_{\mathrm{SM}}$ for (axial-)vector mediators. We also note that LHC exclusion bounds do not depend strongly on the dark matter mass; $\sigma_{\text {collider }}$ refers to the MCFM cross section for a parton $p_{T}>15 \mathrm{GeV}$. 

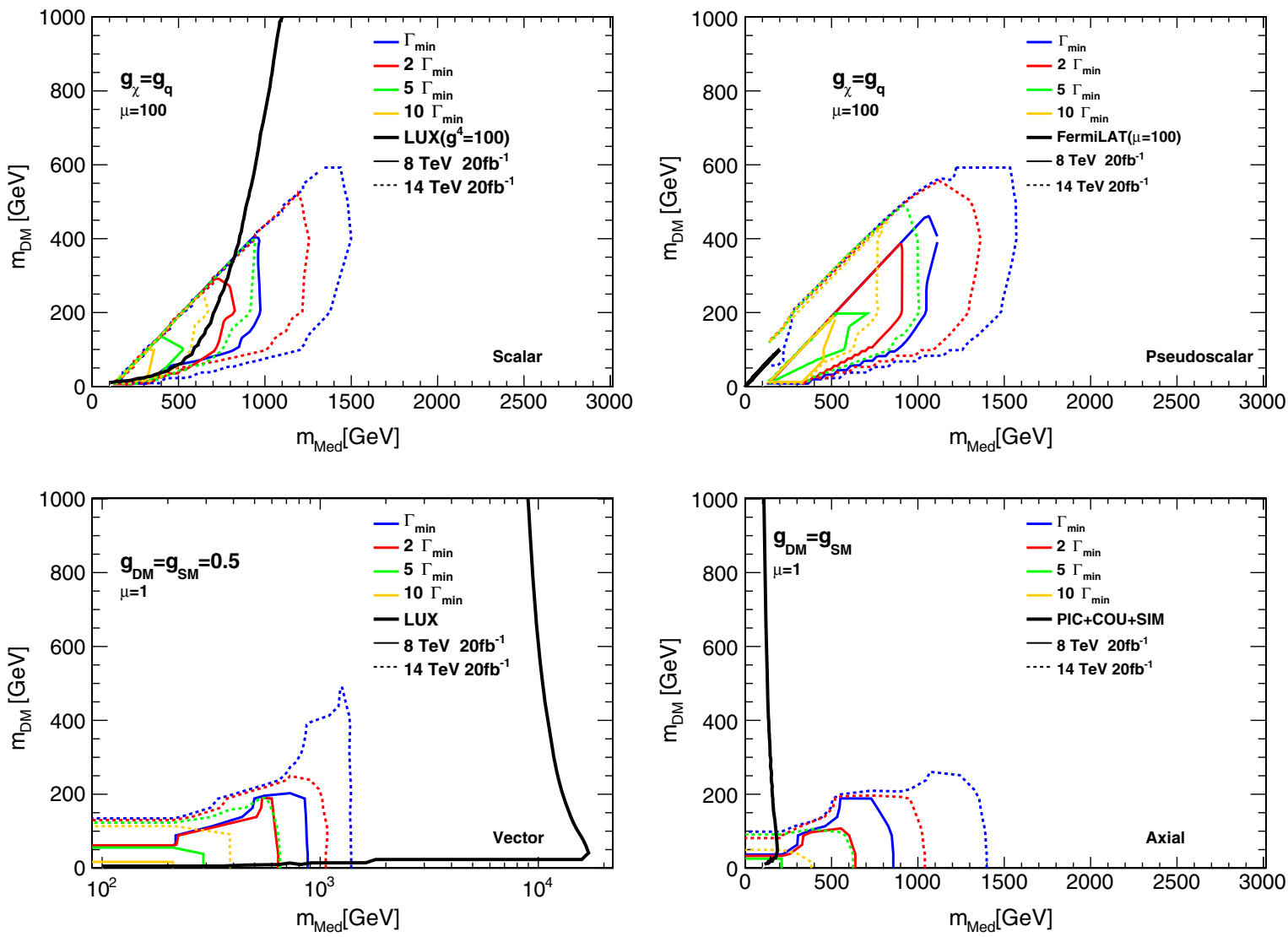

FIG. 6 (color online). $\quad m_{\mathrm{DM}}, m_{\mathrm{MED}}$ exclusion planes for different potential mediators, for a variety of different mediator widths. We show the LHC 8 and the direct detection limits; in addition, we present expected limits at LHC $14 \mathrm{TeV}$ at $20 \mathrm{fb}^{-1}$ as dotted lines. For scalar and pseudoscalar mediators we increased the cross section by a factor of $\mu=100$, as explained in the text. Data for direct detection results (in this and subsequent figures) comes from the LUX [56], PICASSO [63], COUPP [64] and SIMPLE [65] experiments. For the pseudoscalar mediator model on the top-right plot we show the indirect detection limits using FERMI-LAT data [66].

indicate that the excluded couplings and width are smaller than the tested model.

In each plot we show the exclusion contours for different choices of the mediator width $\Gamma_{\mathrm{MED}}$, starting with the minimal width $\Gamma_{\min }$ computed in each model for the given choice of parameters, and then scaling it upwards as $2 \times \Gamma_{\min }, 5 \times \Gamma_{\min }$ and $10 \times \Gamma_{\min }$. We quote this in terms of both a cross section limit and $\mu$. For low values of the mediator mass, we find an exclusion of order unity in $\mu$. Additionally, we observe that increasing the energy from 8 to $14 \mathrm{TeV}$ results in a sizable increase in the limit for heavy $(>1 \mathrm{TeV})$ mediators.

In order to obtain relevant exclusion limits for our simplified models, we must compare the predicted value of the cross section for a given parameter set against the limit set by the LHC (e.g. in Fig. 5). We present the constrained region as a function of the dark matter and mediator mass in Fig. 6.

To highlight the complementarity between the LHC limits and the direct detection (DD) experiments we present them on the same plots in Fig. 6. We stress, however, that the direct comparison between the collider limits on the production of a dark sector particle $\chi$ and the exclusion limits from direct detection and indirect detection (ID) dark matter experiments is only sensible for $\chi$ being the cosmologically stable DM. More generally, if $\chi$ is a representative of the dark sector and is only stable on collider time scales, but not cosmologically, DD and ID bounds are severely diluted relative to the LHC limits in Fig. 6, or even not applicable.

We find that for our vector and axial-vector models described above, the $\mathrm{LHC}$ at $8 \mathrm{TeV}$ can exclude mediator masses of $<1 \mathrm{TeV}$ and dark matter masses of less than around $200 \mathrm{GeV}$. Our $14 \mathrm{TeV} \mathrm{LHC}$ at $20 \mathrm{fb}^{-1}$ projection contours improve these, roughly speaking, by a factor of 2 . Note, however, that the collider limits are strongly dependent on the width of the mediator particle (due to the $\Gamma_{\text {MED }}^{-1}$ scaling of the cross section); if the width is increased the limits become substantially weaker. ${ }^{5}$ For direct detection experiments the limits for axial and vector are very

\footnotetext{
${ }^{5}$ Note that due to the shape of the LHC exclusions for different limits in Fig. 5, this is not a simple $\Gamma_{\text {MED }}^{-1}$ relation.
} 

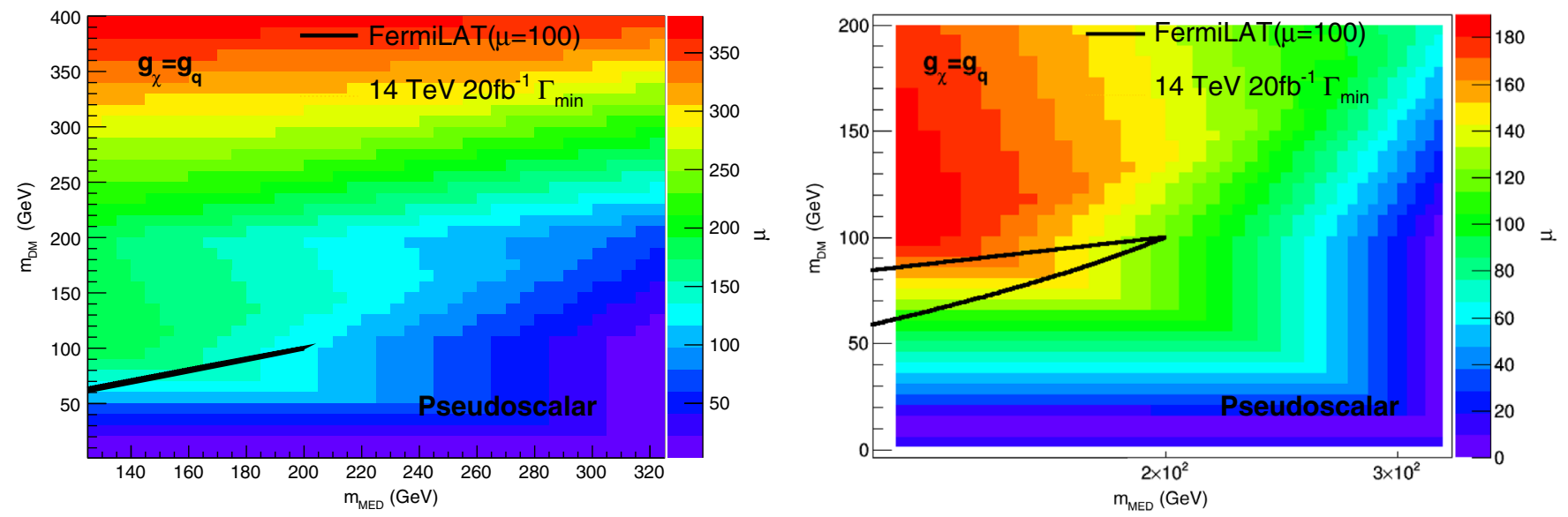

FIG. 7 (color online). Contour plots illustrating the signal enhancement $\mu$-factor for a pseudoscalar mediator to achieve a 90\% C.L. exclusion at $14 \mathrm{TeV}$ LHC. The plot on the right shows exclusion contours for the low DM mass region, cf. top-right plot in Fig. 6.

different, which, as is well established, is due to the spinindependent versus spin-dependent nature of particular processes. For the spin-independent vector scattering, the direct detection experiments cover almost the entire available phase space in the plot (apart from very light DM $<5 \mathrm{GeV}$ scenarios which fall below the threshold energy of the detector). On the other hand, spin-dependent interactions are not as strongly constrained by DD experiments; here constraints are strong for light mediators but fall off quickly for heavier mediators. The complementarity of DD and collider experiments can scarcely be made more apparent than this plot illustrates.

Direct- and indirect-detection experiments are more constrained by the mediator mass than the dark matter mass [cf. Eqs. (16)-(19)], and as a result, are able to exclude a wider range of dark matter masses, at a cost of a smaller constraint on the mediator mass. For the scalar and pseudoscalar case, no collider limit can be set with our choice of $g_{\chi}=g_{q}=1$ - that is to say, experiments cannot presently exclude parameters arising from a model in which both couplings are exactly Higgs-like (for $m_{\mathrm{DM}}=10 \mathrm{GeV}$, at least). From the lower panels of Fig. 5 it is clear that if we increase the signal by a factor of $\mu=100$, then limits can be derived. Note that this scale factor is not completely unmotivated; the width of the mediator for scalar and pseudoscalar mediators in our model is dominated by $t \bar{t}$ decays (in particular for light DM). Therefore, increasing the cross section by a factor of 100 is approximately equivalent to changing $g_{\chi} \rightarrow 10 g_{\chi}$. Since we defined $g_{\mathrm{DM}}=g_{\chi} m_{\mathrm{DM}} / v$, increasing the coupling by a factor of 10 for light DM is completely plausible, for light dark matter, e.g. $m_{\mathrm{DM}} \lesssim 25 \mathrm{GeV}$, such that we remain well within the perturbative regime of $g_{\mathrm{DM}} \lesssim 1$, and is basically equivalent to not requiring the mediator- $\chi$ interaction to scale like a Yukawa coupling. We note that the LUX and FERMI-LAT contours shown on the scalar and pseudoscalar plots of Fig. 6 are obtained with the same rescaling, illustrating the challenge of searching for scalar mediators at all kinds of experiments. Extending the LHC reach to $14 \mathrm{TeV}$ provides more stringent bounds, since the larger center-of-mass energy allows heavier mediators to be probed.

Plots presented in Fig. 7 illustrate the contours of the required $\mu$-factor necessary to enhance the signal for pseudoscalar messenger models to set a $90 \%$ C.L. at $14 \mathrm{TeV}$ LHC assuming the minimal width. The question of whether a parameter point is visible at the LHC depends on the ability to separate signal processes from the background. A better background rejection will boost sensitivity independently of the signal parametrization, and the real analysis sensitivity is likely to improve by a substantial amount. Hence, this figure only serves as a baseline for our current sensitivity. The plot on the right of Fig. 7 zooms into the relatively low DM mass region of the parameter space for pseudoscalar mediators where the data from indirect detection experiments become relevant.

Finally, in Figs. 8 and 9 we show the plots in terms of the spin-dependent and the spin-independent DM-neutron cross sections for a more traditional comparison of collider limits in terms of our simplified models with the limits/ projections from the direct- and indirect-detection experiments computed using cross-section formulas in Sec. III. We compare the results in the $\sigma-m_{\mathrm{MED}}$ and $\sigma-m_{\mathrm{DM}}$ planes. The general pattern of Fig. 6 is reproduced, with spin-independent results from LUX providing the strongest bounds; for the axial and scalar cases the example we have chosen to illustrate is for a mediator which is too heavy to be efficiently probed at DD experiments, resulting in stronger bounds from the LHC.

In Fig. 10, we show the spin-dependent cross-section limits deduced from the LHC projections and FERMI-LAT now for a light $125 \mathrm{GeV}$ mediator. 

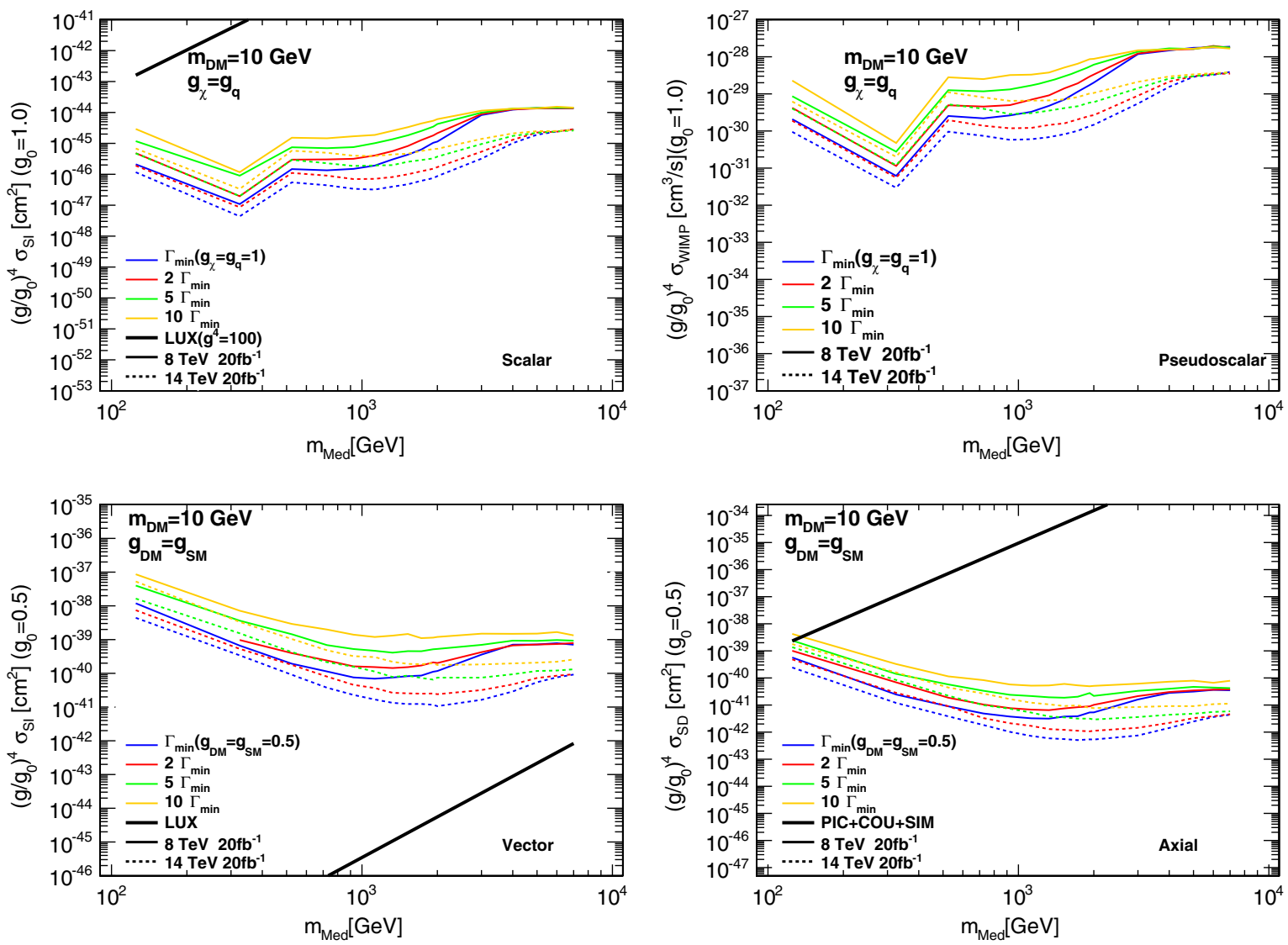

FIG. 8 (color online). Cross-section limits (projections) in terms of those reported by DD experiments; we show exclusions in terms of the spin-dependent and spin-independent cross sections against mediator mass for scalar, vector and axial-vector mediators. For the pseudoscalar mediator we plot the DM pair annihilation cross section. The corresponding limit from indirect detection experiments is too weak to show for the pseudoscalar messenger.

\section{EFFECTS OF HEAVY NEW PHYSICS ON MEDIATOR PRODUCTION}

In this section we investigate potential BSM effects which may alter the production of the dark sector mediator. In particular we focus on additional heavy degrees of freedom, which are charged under $S U\left(N_{c}\right)$. Since we assume that these new degrees of freedom are heavy, we can work in the limit in which the new states are integrated out (however, we stress that the mediator remains a propagating particle). This is achieved by including the following interaction in our simplified model Lagrangian in Eq. (1):

$$
\mathcal{L}_{\mathrm{EFT}}=g_{g} \frac{\alpha_{s}}{12 \pi v} S \operatorname{Tr}\left(G^{\mu \nu} G_{\mu \nu}\right) .
$$

For simplicity we have focused on the scalar mediator and parameterized the Lagrangian in terms of a rescaled Higgs EFT dimension-5 operator (in which the rescaling factor is $g_{q}$ ). Our extended simplified model now has an additional parameter $g_{g}$, resulting in a total of six free parameters.

In order to make predictions for the resulting model, we need to extend the existing implementation of this process in MCFM [6], which is based upon modified matrix elements for Higgs production (computed originally in Ref. [76]). The inclusion of Eq. (21) in the Lagrangian results in a new term which interferes with the top loop contribution at the amplitude level. Accordingly, we have recomputed the production amplitude $g g \rightarrow g+S$ and $q \bar{q} \rightarrow g+S$ in terms of helicity amplitudes. The results for these amplitudes, which to the best of our knowledge, have not been reported elsewhere, are included in the Appendix. Representative Feynman diagrams from this extended model are the first two diagrams in Fig. 1, with the first representing the new BSM contribution assumed to be induced by heavy colored particles.

In Fig. 11 we present the cross-section ratio $\sigma\left(g_{g} \neq 0\right) /$ $\sigma\left(g_{g}=0\right)$ computed in the extended simplified model. Cross sections are obtained using the basic cuts described in Sec. IV, i.e. we require $p_{T, j_{1}} \geq 110 \mathrm{GeV}$ and $|\eta|<2.4$. Since these are LO cross sections, an equivalent cut of $110 \mathrm{GeV}$ is imposed upon the MET. Cross sections are presented for the $14 \mathrm{TeV}$ LHC, and CTEQ6L1 [77] PDF sets have been used; the renormalization and factorization scale are set to $\mu=m_{\chi \bar{\chi}}$. Parametrically, we expect a 

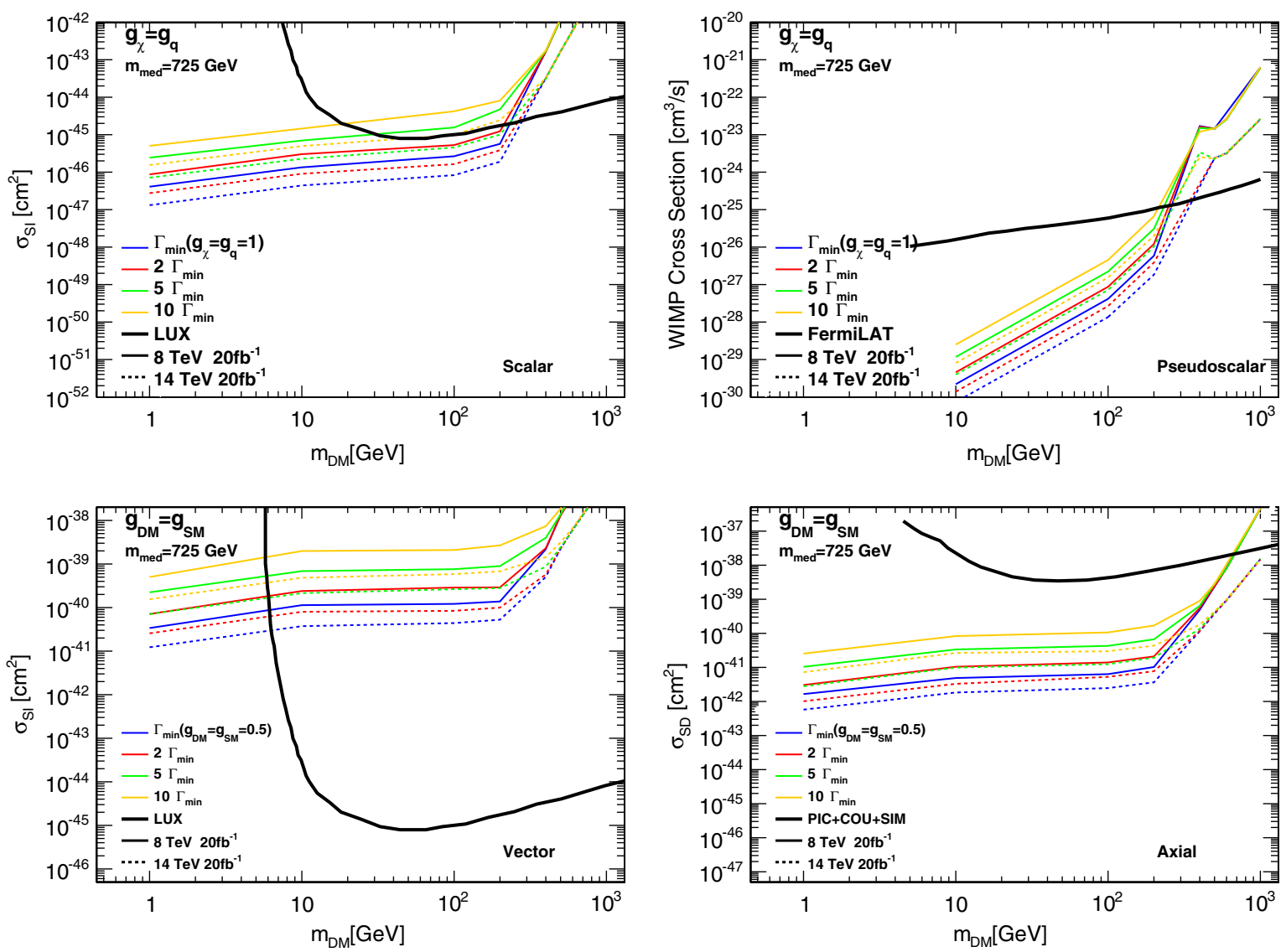

FIG. 9 (color online). Exclusion contours for the spin-dependent and spin-independent cross sections as in Fig. 8, now plotted as functions of the dark matter mass. For the pseudoscalar mediator model, we show the indirect detection limits (using FERMI-LAT data [66]). For the pseudoscalar we show 95\% C.L. exclusion limits, while we show limits at 90\% C.L. for the other mediators.

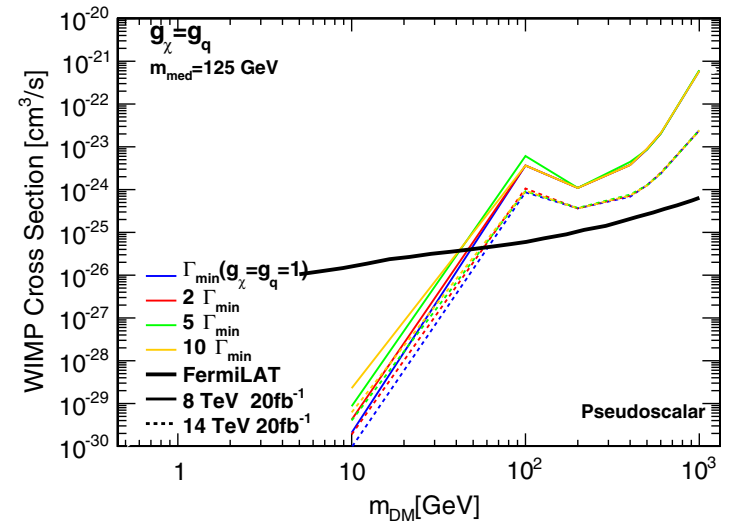

FIG. 10 (color online). The spin-independent cross section as in top-right Fig. 9 for a $125 \mathrm{GeV}$ pseudoscalar mediator mass.

prediction which is of the form $a_{0}+a_{1} g_{g}+a_{2} g_{g}^{2}$, and we expect the curves presented in Fig. 11 illustrate this functionality. We have chosen to illustrate two benchmark points, which correspond to a situation in which the (Yukawa-adjusted) mediator-top and mediator-DM coupling are set equal $\left(g_{t}=g_{\chi}=1\right)$, and a second scenario in which the coupling to the top is damped $g_{t}=g_{\chi} / 10=0.1$.
Clearly, in the second scenario we expect a significantly bigger contribution from the heavy particles in the loop, since the top is suppressed. This is illustrated in Fig. 11, for which we see enhancements of order 1-3 for the democratic model, and 10-100 for the top-suppressed model. The second case is analogous to the situation in the production of a SM Higgs, in which the propagating $b$-quark loops are suppressed relative to the top-quark contribution, which can be evaluated in the heavy top limit. We note that the shapes of the curves in Fig. 11 are dominantly due to the relative importance of $g_{t}$ compared to $g_{g}$, and the mediator mass. The dependence on $g_{\chi}$ cancels in the ratio, as does the decay matrix element to DM; therefore, although we chose a benchmark mass of $10 \mathrm{GeV}$, Fig. 11 would not be changed if a different $m_{\mathrm{DM}}$ were used.

Under the assumption that the scalar mediator proceeds through a portal interaction with the Higgs boson, $g_{g}$ plays the role of a mixing angle-if $g_{g}$ becomes large, significant deviations from the SM Higgs should be expected, and have not been observed. If instead the scalar is allowed to couple to the heavy degrees of freedom in an arbitrary way, then we interpret $g_{g}$ as a rescaling factor which relates the 

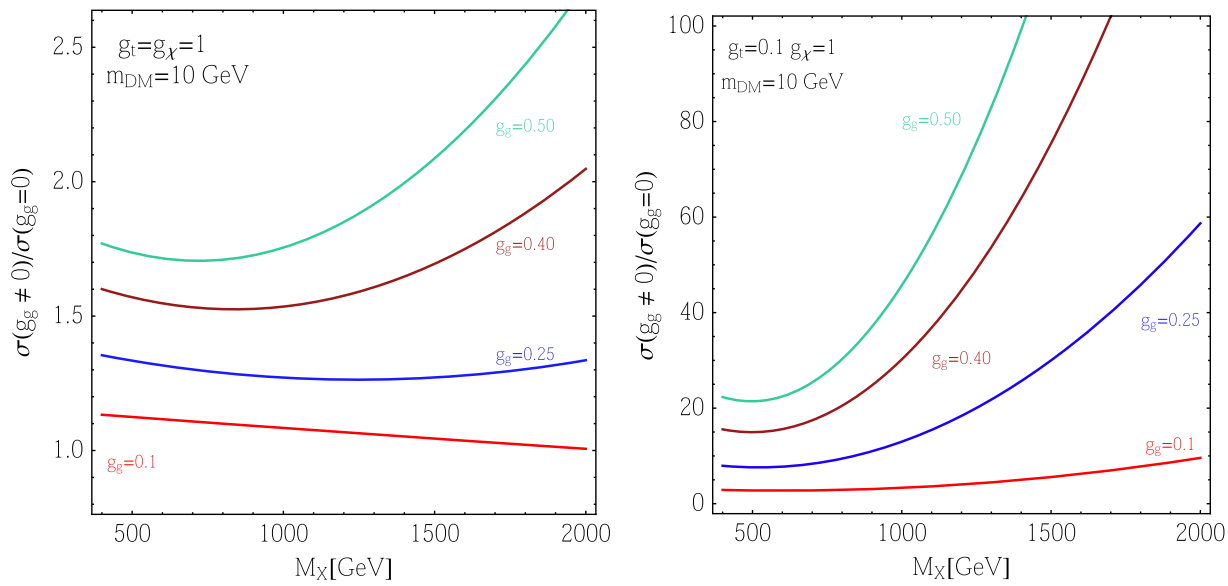

FIG. 11 (color online). Cross-section ratios describing the impact of the five-dimensional contact interaction between gluons and the scalar mediator. The ratio is computed for fixed $g_{\chi}=1$, and the plot on the left also sets $g_{t}=1$, while on the right the top-mediator coupling is weakened to $g_{t}=0.1$. In both instances the width is evaluated as the minimal width. The dark matter mass is fixed at $m_{\mathrm{DM}}=100 \mathrm{GeV}$; the mediator mass is varied.

coupling and mass scale of the dark sector to that of the EW scale, i.e.

$$
g_{g} \sim \frac{g_{\mathrm{NP}}}{\Lambda_{\mathrm{NP}}} \frac{v}{g_{w}} .
$$

In order for the EFT to be valid, we need to ensure that the kinematic distributions are probed at scales less than $\Lambda_{\mathrm{NP}}$. We present the differential distribution for the missing transverse momentum in Fig. 12. At $14 \mathrm{TeV}$ the tail of the distribution probes scales of around a few $\mathrm{TeV}$; setting $\Lambda_{\mathrm{NP}}=2 \mathrm{TeV}$ and assuming $g_{\mathrm{NP}}$ is $\mathcal{O}(1)$, we see that the maximum $g_{g}$ which can be safely probed at the $14 \mathrm{TeV}$ LHC is around $g_{g}<0.3$. Figure 12 illustrates the usual result - that higher-dimensional operators are relatively less suppressed at high energies compared to their fourdimensional counterparts. As a result, the impact of the $g_{q}$ pieces can be reinterpreted as a momentum-dependent form factor which modifies the four-dimensional Lagrangian.

The results presented in this section suggest that, should a propagating resonance be found in the monojet channel at the LHC, coupling constraints on loop-induced heavy sector particles can be investigated, of which values approximately $g_{g}<0.3$ correspond to theories in which the EFT prescription is viable. These constraints may shed light on extended sectors in the BSM theory which contain heavy colored particles. In addition, if run II searches based on the simplified models defined in Sec. II lead to null results, then one can also test models in which the scalar mediator and putative dark matter particles are light, but
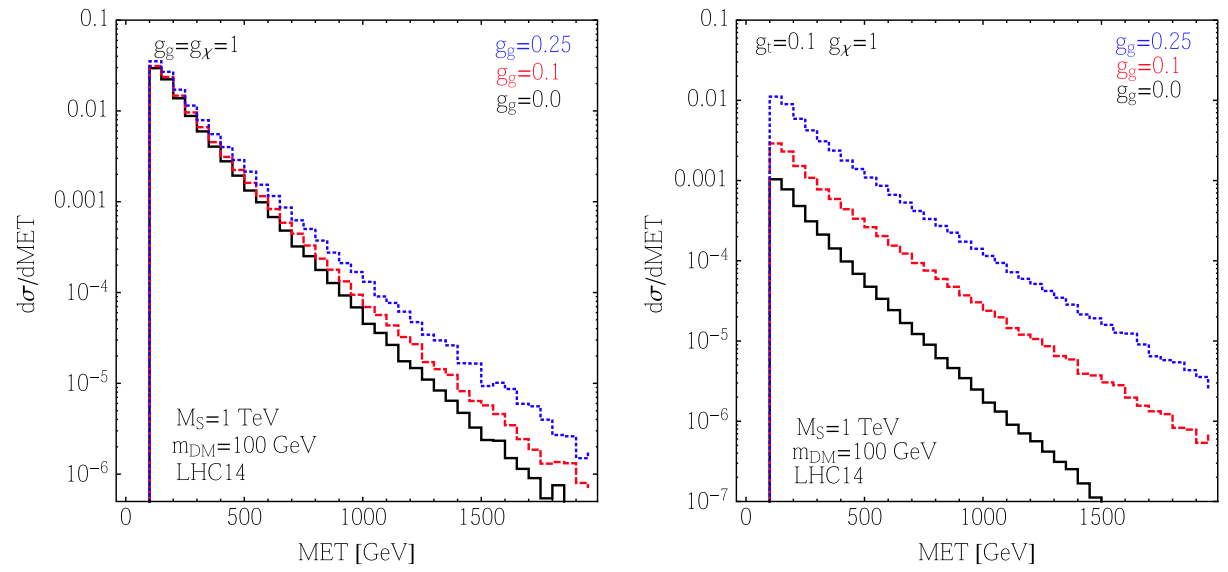

FIG. 12 (color online). The missing transverse energy (MET) differential distribution showing parton-level predictions obtained using several choices of $g_{g}$ for a benchmark scenario in which the dark matter particles are $100 \mathrm{GeV}$ and the mediator is $1 \mathrm{TeV}$. The plot on the left corresponds to the democratic choice of mediator-top and mediator-DM coupling $\left(g_{t}=g_{\chi}=1\right)$, while that on the right corresponds to the case in which $g_{t}=g_{\chi} / 10=0.1$. 
only couple to the SM through a heavy colored messenger. These instances correspond exactly to the situation in which $g_{g}$ is nonzero, but $g_{t} \ll g_{g}, g_{\chi}$. In theories with this coupling structure the EFT becomes the dominant production model, and although at the cost of an additional parameter, $g_{g}$ should be included in the simplified model.

\section{CONCLUSIONS}

We have defined benchmark or simplified models for dark particle searches for the cases of scalar, pseudoscalar, vector and axial-vector mediators between the SM and dark sectors. These models are defined by the interaction in Eqs. (1)-(4) and (21). Apart from the choice of mediator type, these models are characterized in our approach by the following free parameters:

(1) Mediator mass $m_{\mathrm{MED}}$.

(2) Mediator width $\Gamma_{\text {MED }}$.

(3) Dark matter mass $m_{\mathrm{DM}}$.

(4) Effective coupling parameter $g_{q} \cdot g_{\chi}$ for scalar and pseudoscalar mediators (5), and $g_{\mathrm{SM}} \cdot g_{\mathrm{DM}}$ for axialvector and vector mediators.

In our examples here, we chose to study democratic scenarios in which the couplings in the dark sector and SM were equal; although this need not be the case, this reduces the degrees of freedom from five to four. We have implemented simplified models based on these parameters into a fully flexible (and public) Monte Carlo code, MCFM. We used MCFM to generate signal events, which were processed through event and detector simulation for the 8 and $14 \mathrm{TeV}$ LHC. We were then able to use a recent CMS analysis to study benchmark points in our simplified models, producing cross section limits, limits in the mediator-dark matter mass plane, and cross section versus mass limits.

The introduction of the simplified models greatly increases the number of free parameters which enter searches at the LHC. Previous iterations of experimental results focused on the regime in which the mediator is assumed to be heavy, such that an effective field theory description is valid. In this setup one has to constrain one parameter per operator, the Wilson coefficient $C_{i}$. However, at the LHC energies many interesting scenarios occur in which the mediator can be produced on shell, which makes the introduction of simplified models a useful tool. Future iterations of LHC searches have the harder task of presenting results in this five-dimensional plane.

If the simplified model is extended to include colored degrees of freedom, modifications can occur in the production of the mediating particle. This is particularly relevant for the scalar and pseudoscalar cases, which proceed at the oneloop level. Heavy colored physics can couple directly to the mediator and result in an additional five-dimensional contribution to the Lagrangian. We investigated the impact of this term for the scalar mediated case and found that if the mediator-top coupling is damped, then significant contributions to the production cross section can arise from these terms. This is analogous to the situation in the SM, in which the light bottom quark loops are much smaller than the top quark loops (which can be treated in an effective field theory approach).

We find that limitations of direct- and indirect-detection experiments, i.e. velocity suppression and loop-suppressed couplings to Standard Model particles, can be overcome by LHC searches. Thus, the LHC provides a complementary coverage of the dark sector parameter space with respect to low-energy experiments. Importantly, if the invisible particle is not stable on cosmological time scales, the LHC can be the only experiment to probe the dark sector.

The search for dark matter whether in direct, indirect, or collider experiments represents one of the most fascinating and challenging goals for physics this century. The LHC is about to enter a new era with the start of run II, and with this the evolution of the LHC searches to incorporate more complete UV models and include the region in which EFT assumptions break down is a natural progression. The simplified models we have discussed here provide a pathway to achieving this.

\section{ACKNOWLEDGMENTS}

We would like to thank Brian Batell, Oliver Buchmueller, Albert De Roeck, Patrick Fox, Christopher McCabe, Tim Tait and Christopher Wallace for valuable discussions. The research of V. V. K. and M. S. is supported by STFC through the IPPP grant and for V. V. K. by the Wolfson Foundation and Royal Society.

Note added.-Recently, Ref. [10] was posted on arXiv, which also considers scalar and pseudoscalar mediators in the gluon fusion channel and treats $\Gamma_{\mathrm{MED}}$ as a free parameter.

\section{APPENDIX: AMPLITUDES FOR $g g \rightarrow S+g$ AND $q \bar{q} \rightarrow S+g$}

In this section we present the helicity amplitudes for $g g \rightarrow S+g$ and $q \bar{q} \rightarrow S+g$ in both the full and effective field theories. Helicity amplitudes are defined in terms of $u_{ \pm}\left(k_{i}\right)$ where $u$ represents a Weyl spinor of momentum $k_{i}$, with either positive or negative helicity. Basic spinor products are then defined as

$$
\begin{aligned}
& \langle i j\rangle=\left\langle i^{-} \mid j^{+}\right\rangle=\bar{u}_{-}\left(k_{i}\right) u_{+}\left(k_{j}\right), \\
& {[i j]=\left\langle i^{+} \mid j^{-}\right\rangle=\bar{u}_{+}\left(k_{i}\right) u_{-}\left(k_{j}\right) .}
\end{aligned}
$$

Kinematic invariants are constructed from products of the above quantities:

$$
\langle i j\rangle[j i]=2 k_{i} k_{j}=s_{i j} .
$$

Spinor strings are defined as follows: 


$$
\left\langle i\left|P_{k l}\right| j\right]=\langle i|(k+l)| j]=\langle i k\rangle[k l]+\langle i l\rangle[l j] .
$$

We begin by presenting the $g g \rightarrow S+g$ amplitude with a propagating fermion of mass $m_{f}$ in the loop. We decompose the amplitude in terms of a kinematic primitive amplitude and normalization factors as follows:

$$
A_{4}\left(S, 1_{g}^{h_{1}}, 2_{g}^{h_{2}}, 3_{g}^{h_{3}}\right)=N_{c}\left(N_{c}^{2}-1\right) \frac{g_{f} m_{f}^{2} g_{w}}{16 \pi^{2} M_{W}}\left(\frac{g_{s}}{\sqrt{2}}\right)^{3} \mathcal{A}\left(S, 1_{g}^{h_{1}}, 2_{g}^{h_{2}}, 3_{g}^{h_{3}}\right)
$$

Of the possible helicity orderings, two can be chosen $(+++,-++)$ from which all remaining amplitudes can be obtained from conjugation and Bose symmetries. The +++ amplitude is given in terms of the following box $\left(D_{i}\right)$ and triangle $\left(C_{i}\right)$ scalar integrals:

$$
\begin{aligned}
\mathcal{A}\left(S, 1_{g}^{+}, 2_{g}^{+}, 3_{g}^{+}\right)= & \left(\frac{[21][31]\left(4 m_{f}^{2}-s_{123}\right)}{\langle 23\rangle} D_{1}\left(s_{13}, s_{12}, m_{f}^{2}\right)+\frac{s_{12}+s_{13}}{\langle 12\rangle\langle 13\rangle\langle 23\rangle}\left(4 m_{f}^{2}-s_{123}\right) C_{1}\left(s_{23}, s_{123}, m_{f}^{2}\right)\right. \\
& +\{1 \leftrightarrow 3\}+\{1 \leftrightarrow 2\})+\frac{s_{123}}{\langle 12\rangle\langle 13\rangle\langle 23\rangle} .
\end{aligned}
$$

Here $D_{1}\left(s, t, m_{f}^{2}\right)$ represents a box integral with a single off-shell leg $\left(s_{123}\right)$, which is specified completely by $s$ - and $t$-channel invariants, and $C_{1}\left(s, t, m_{f}^{2}\right)$ represents the triangle integral with two legs $s$ and $t$ off shell. The second helicity amplitude required also contains bubble integrals $B_{i}$ :

$$
\begin{aligned}
\mathcal{A}\left(S, 1_{g}^{+}, 2_{g}^{+}, 3_{g}^{+}\right)= & -\frac{\langle 12\rangle\langle 13\rangle}{\langle 23\rangle^{3}}\left(4 s_{12} s_{13}+12 m_{f}^{2}-s_{23}^{2}\right) D_{1}\left(s_{13}, s_{12}, m_{f}^{2}\right)+2 \frac{\left(s_{12}+s_{13}\right)[32]\left(4 m_{f}^{2}-s_{23}\right)}{\langle 23\rangle^{2}[21][31]} C_{1}\left(s_{23}, s_{123}, m_{f}^{2}\right) \\
& +\left\{\frac{\langle 12\rangle[23]^{2}\left(4 m_{f}^{2}-s_{23}\right)}{\langle 23\rangle[31]} D_{1}\left(s_{12}, s_{23}, m_{f}^{2}\right)+\frac{4\langle 12\rangle\langle 13\rangle s_{13}}{\langle 23\rangle} C_{2}\left(s_{13}, m_{f}^{2}\right)\right. \\
& +\left(-4 \frac{\langle 12\rangle\langle 13\rangle\left\langle 3\left|P_{12}\right| 3\right]}{\langle 23\rangle^{3}}+2 \frac{s_{13}\langle 13\rangle[32]^{2}}{\langle 23\rangle[21]\left\langle 3\left|P_{12}\right| 3\right]}-2 \frac{s_{23}[23]^{3}}{[21][31]\left\langle 3\left|P_{12}\right| 3\right]}\right. \\
& \left.-4 m_{f}^{2}\left(2 \frac{s_{13}\langle 13\rangle[32]}{\langle 23\rangle^{2}[21]\left\langle 3\left|P_{12}\right| 3\right]}+4 \frac{\langle 13\rangle[32]^{3}}{\langle 23\rangle[21]\left\langle 3\left|P_{12}\right| 3\right]}+2 \frac{[32]^{3}}{\left\langle 3\left|P_{12}\right| 3\right][21][31]}\right)\right) C_{1}\left(s_{12}, s_{123}, m_{f}^{2}\right) \\
& \left.+8 \frac{\langle 12\rangle\langle 13\rangle[32]\left(s_{13}+2 s_{123}\right)}{\langle 23\rangle^{2}\left\langle 3\left|P_{12}\right| 3\right]^{2}}\left(B_{1}\left(s_{12}, m_{f}^{2}\right)-B_{1}\left(s_{123}, m_{f}^{2}\right)\right)+\{2 \leftrightarrow 3\}\right\} \\
& -\frac{[32]^{2}\left(s_{12} s_{13}+s_{23}^{2}\right)}{\langle 23\rangle[21][31]\left\langle 3\left|P_{12}\right| 3\right]\left\langle 2\left|P_{13}\right| 2\right]} .
\end{aligned}
$$

Here we have introduced additional triangle integrals $C_{2}\left(s, m_{f}^{2}\right)$ which represent topologies with one off-shell leg $(s)$ and bubble integrals $B_{1}\left(s, m_{f}^{2}\right)$ which depend on a scale $s$. The basis integrals in the above equations can be easily evaluated using public packages; our implementation in MCFM uses QCDLoop [78].

The amplitude for $q \bar{q} \rightarrow S+g$ can be written as follows:

$$
A_{4}\left(S, 1_{\bar{q}}^{h_{1}}, 2_{q}^{-h_{1}}, 3_{g}^{h_{3}}\right)=\left(N_{c}^{2}-1\right) \frac{g_{f} m_{f}^{2} g_{w} g_{s}^{3}}{16 \pi^{2} M_{W}} \mathcal{A}\left(S, 1_{\bar{q}}^{h_{1}}, 2_{q}^{-h_{1}}, 3_{g}^{h_{3}}\right)
$$

Here the primitive amplitude can be defined in terms of one helicity configuration $(-++)$, with all other configurations obtainable from line reversal and conjugation symmetries. Our primitive amplitude is 
$\mathcal{A}\left(1_{\bar{q}}^{-}, 2_{q}^{+}, 3+\right)=2 \frac{[23]^{2}}{[21]}\left(\left\langle 3\left|P_{12}\right| 3\right]-4 m_{f}^{2}\right) C_{1}\left(s_{12}, s_{123}, m_{f}^{2}\right)-4 \frac{\langle 12\rangle[32]^{2}}{\left\langle 3\left|P_{12}\right| 3\right]^{2}}\left(B_{1}\left(s_{12}, m_{f}^{2}\right)-B_{1}\left(s_{123}, m_{f}^{2}\right)+4 \frac{[32]^{2}}{[21]\left\langle 3\left|P_{12}\right| 3\right]}\right.$.

In addition to the amplitudes presented above, we will also need the $m_{f} \rightarrow \infty$ limit, which corresponds to amplitudes computed in the effective field theory. These amplitudes have been computed in Ref. [79] and have the following form:

$$
\begin{gathered}
\mathcal{A}_{m_{f} \rightarrow \infty}\left(S, 1_{g}^{+}, 2_{g}^{+}, 3_{g}^{+}\right)=\frac{s_{123}^{2}}{\langle 12\rangle\langle 23\rangle\langle 31\rangle}, \\
\mathcal{A}_{m_{f} \rightarrow \infty}\left(S, 1_{g}^{+}, 2_{g}^{-}, 3_{g}^{-}\right)=\frac{\langle 23\rangle^{2}}{\langle 12\rangle\langle 31\rangle}
\end{gathered}
$$

[1] D. Alves et al. (LHC New Physics Working Group), Simplified models for LHC new physics searches, J. Phys. G 39, 105005 (2012).

[2] J. Abdallah, A. Ashkenazi, A. Boveia, G. Busoni, A. De Simone, C. Doglioni, A. Efrati, E. Etzion et al., Simplified models for dark matter and missing energy searches at the LHC, arXiv:1409.2893.

[3] S. Malik, C. McCabe, H. Araujo, A. Belyaev, C. Boehm, J. Brooke, O. Buchmueller, G. Davies et al., Interplay and characterization of dark matter searches at colliders and in direct detection experiments, arXiv:1409.4075.

[4] P. J. Fox, R. Harnik, J. Kopp, and Y. Tsai, LEP shines light on dark matter, Phys. Rev. D 84, 014028 (2011).

[5] P. J. Fox, R. Harnik, J. Kopp, and Y. Tsai, Missing energy signatures of dark matter at the LHC, Phys. Rev. D 85, 056011 (2012).

[6] P. J. Fox and C. Williams, Next-to-leading order predictions for dark matter production at hadron colliders, Phys. Rev. D 87, 054030 (2013).

[7] H. An, X. Ji, and L. T. Wang, Light dark matter and $Z^{\prime}$ dark force at colliders, J. High Energy Phys. 07 (2012) 182.

[8] O. Buchmueller, M. J. Dolan, and C. McCabe, Beyond effective field theory for dark matter searches at the LHC, J. High Energy Phys. 01 (2014) 025.

[9] O. Buchmueller, M. J. Dolan, S. A. Malik, and C. McCabe, Characterising dark matter searches at colliders and direct detection experiments: Vector mediators, J. High Energy Phys. 01 (2015) 037.

[10] M. R. Buckley, D. Feld, and D. Goncalves, Scalar simplified models for dark matter, Phys. Rev. D 91, 015017 (2015).

[11] C. Englert, J. Jaeckel, E. Re, and M. Spannowsky, Evasive Higgs maneuvers at the LHC, Phys. Rev. D 85, 035008 (2012).

[12] G. Aad et al. (ATLAS Collaboration), Search for new phenomena with the monojet and missing transverse momentum signature using the ATLAS detector in $\sqrt{s}=7 \mathrm{TeV}$ proton-proton collisions, Phys. Lett. B 705, 294 (2011).
[13] J. M. Campbell and R. K. Ellis, An update on vector boson pair production at hadron colliders, Phys. Rev. D 60, 113006 (1999).

[14] J. M. Campbell, R. K. Ellis, and C. Williams, Vector boson pair production at the LHC, J. High Energy Phys. 07 (2011) 018.

[15] J. M. Campbell, R. K. Ellis, and C. Williams, Vector boson pair production at the LHC, MCFM website, http://mcfm .fnal.gov.

[16] U. Haisch, F. Kahlhoefer, and E. Re, QCD effects in monojet searches for dark matter, J. High Energy Phys. 12 (2013) 007.

[17] T. Aaltonen et al. (CDF Collaboration), A Search for Dark Matter in Events with One Jet and Missing Transverse Energy in $p \bar{p}$ Collisions at $\sqrt{s}=1.96 \mathrm{TeV}$, Phys. Rev. Lett. 108, 211804 (2012).

[18] S. Chatrchyan et al. (CMS Collaboration), Search for dark matter and large extra dimensions in monojet events in $p p$ collisions at $\sqrt{s}=7 \mathrm{TeV}$, J. High Energy Phys. 09 (2012) 094.

[19] ATLAS Collaboration, Report No. ATLAS-CONF-2012-084.

[20] V. Khachatryan et al. (CMS Collaboration), Search for dark matter, extra dimensions, and unparticles in monojet events in proton-proton collisions at $\sqrt{s}=8 \mathrm{TeV}$, arXiv:1408.3583.

[21] E. Diehl (ATLAS Collaboration), The search for dark matter using monojets and monophotons with the ATLAS detector, AIP Conf. Proc. 1604, 324 (2014).

[22] V. D. Barger, K. Hagiwara, J. Woodside, and W. Y. Keung, Possible Supersymmetry Scenario for $p \bar{p}$ Collider Monojet Events and Unaccompanied "Photon" Events, Phys. Rev. Lett. 53, 641 (1984).

[23] E. W. N. Glover, A. D. Martin, and M. R. Pennington, Collider monojets as a signature of new dynamics, Phys. Lett. 153B, 330 (1985).

[24] L. J. Hall and A. E. Nelson, Heavy gluons and monojets, Phys. Lett. 153B, 430 (1985). 
[25] M. Beltran, D. Hooper, E. W. Kolb, Z. A. C. Krusberg, and T. M. P. Tait, Maverick dark matter at colliders, J. High Energy Phys. 09 (2010) 037.

[26] V. M. Abazov et al. (D0 Collaboration), Search for Large Extra Dimensions in the Monojet + Missing $E_{T}$ Channel at D0, Phys. Rev. Lett. 90, 251802 (2003).

[27] L. J. Hall and J. Polchinski, Implications of supersymmetric origins for monojets, Phys. Lett. 152B, 335 (1985).

[28] J. F. Gunion and S. Mrenna, Probing models with near degeneracy of the chargino and LSP at a linear $e^{+} e^{-}$ collider, Phys. Rev. D 64, 075002 (2001).

[29] H. K. Dreiner, M. Kramer, and J. Tattersall, How low can SUSY go? Matching, monojets and compressed spectra, Europhys. Lett. 99, 61001 (2012).

[30] U. Haisch, F. Kahlhoefer, and J. Unwin, The impact of heavy-quark loops on LHC dark matter searches, J. High Energy Phys. 07 (2013) 125.

[31] J. L. Feng, S. Su, and F. Takayama, Lower Limit on Dark Matter Production at the Large Hadron Collider, Phys. Rev. Lett. 96, 151802 (2006).

[32] Q. H. Cao, C. R. Chen, C.S. Li, and H. Zhang, Effective dark matter model: Relic density, CDMS II, Fermi LAT and LHC, J. High Energy Phys. 08 (2011) 018.

[33] J. Goodman, M. Ibe, A. Rajaraman, W. Shepherd, T. M. P. Tait, and H. B. Yu, Constraints on light Majorana dark Matter from colliders, Phys. Lett. B 695, 185 (2011).

[34] J. Goodman, M. Ibe, A. Rajaraman, W. Shepherd, T. M. P. Tait, and H. B. Yu, Constraints on dark matter from colliders, Phys. Rev. D 82, 116010 (2010).

[35] C. Englert and M. Spannowsky, Effective theories and measurements at colliders, Phys. Lett. B 740, 8 (2015).

[36] G. Busoni, A. De Simone, E. Morgante, and A. Riotto, On the validity of the effective field theory for dark matter searches at the LHC, Phys. Lett. B 728, 412 (2014).

[37] M. Papucci, A. Vichi, and K. M. Zurek, Monojet versus rest of the world I: t-channel models, J. High Energy Phys. 11 (2014) 024.

[38] G. Busoni, A. De Simone, J. Gramling, E. Morgante, and A. Riotto, On the validity of the effective field theory for dark matter searches at the LHC, part II: Complete Analysis for the $s$-channel, J. Cosmol. Astropart. Phys. 06 (2014) 060.

[39] U. Haisch, A. Hibbs, and E. Re, Determining the structure of dark-matter couplings at the LHC, Phys. Rev. D 89, 034009 (2014).

[40] A. Alves, S. Profumo, and F. S. Queiroz, The dark $Z^{\prime}$ portal: Direct, indirect and collider searches, J. High Energy Phys. 04 (2014) 063.

[41] G. Arcadi, Y. Mambrini, M. H. G. Tytgat, and B. Zaldivar, Invisible $Z^{\prime}$ and dark matter: LHC vs LUX constraints, J. High Energy Phys. 03 (2014) 134.

[42] F. Richard, G. Arcadi, and Y. Mambrini, Search for dark matter at colliders, arXiv:1411.0088.

[43] G. D’Ambrosio, G. F. Giudice, G. Isidori, and A. Strumia, Minimal flavor violation: An effective field theory approach, Nucl. Phys. B645, 155 (2002).

[44] C. Englert, J. Jaeckel, V. V. Khoze, and M. Spannowsky, Emergence of the electroweak scale through the Higgs portal, J. High Energy Phys. 04 (2013) 060.

[45] C. Englert, T. Plehn, D. Zerwas, and P. M. Zerwas, Exploring the Higgs portal, Phys. Lett. B 703, 298 (2011).
[46] V. V. Khoze, Inflation and dark matter in the Higgs portal of classically scale invariant Standard Model, J. High Energy Phys. 11 (2013) 215.

[47] T. Hambye and A. Strumia, Dynamical generation of the weak and dark matter scale, Phys. Rev. D 88, 055022 (2013).

[48] V. V. Khoze, C. McCabe, and G. Ro, Higgs vacuum stability from the dark matter portal, J. High Energy Phys. 08 (2014) 026.

[49] W. Altmannshofer, W. A. Bardeen, M. Bauer, M. Carena, and J. D. Lykken, Light dark matter, naturalness, and the radiative origin of the electroweak scale, J. High Energy Phys. 01 (2015) 032.

[50] M. Heikinheimo, A. Racioppi, M. Raidal, C. Spethmann, and K. Tuominen, Dark supersymmetry, Nucl. Phys. B876, 201 (2013).

[51] E. Gabrielli, M. Heikinheimo, K. Kannike, A. Racioppi, M. Raidal, and C. Spethmann, Towards completing the Standard Model: Vacuum stability, EWSB and dark matter, Phys. Rev. D 89, 015017 (2014).

[52] M. Duerr, P. Fileviez Perez, and M. B. Wise, Gauge Theory for Baryon and Lepton Numbers with Leptoquarks, Phys. Rev. Lett. 110, 231801 (2013).

[53] M. Duerr and P. Fileviez Perez, Baryonic dark matter, Phys. Lett. B 732, 101 (2014).

[54] S. R. Coleman and E. J. Weinberg, Radiative corrections as the origin of spontaneous symmetry breaking, Phys. Rev. D 7, 1888 (1973).

[55] D. S. Akerib et al. (LUX Collaboration), The Large Underground Xenon (LUX) experiment, Nucl. Instrum. Methods Phys. Res., Sect. A 704, 111 (2013).

[56] D. S. Akerib et al. (LUX Collaboration), First Results from the LUX Dark Matter Experiment at the Sanford Underground Research Facility, Phys. Rev. Lett. 112, 091303 (2014).

[57] M. Szydagis et al. (LUX Collaboration), A detailed look at the first results from the Large Underground Xenon (LUX) dark matter experiment, arXiv:1402.3731.

[58] A. Kurylov and M. Kamionkowski, Generalized analysis of weakly interacting massive particle searches, Phys. Rev. D 69, 063503 (2004).

[59] J. Hisano, K. Ishiwata, and N. Nagata, Gluon contribution to the dark matter direct detection, Phys. Rev. D 82, 115007 (2010).

[60] K. Cheung, C. T. Lu, P. Y. Tseng, and T. C. Yuan, Collider constraints on the dark matter interpretation of the CDMS II results, arXiv:1308.0067.

[61] H. Y. Cheng and C.W. Chiang, Revisiting scalar and pseudoscalar couplings with nucleons, J. High Energy Phys. 07 (2012) 009.

[62] K. R. Dienes, J. Kumar, B. Thomas, and D. Yaylali, Overcoming velocity suppression in dark-matter direct-detection experiments, Phys. Rev. D 90, 015012 (2014).

[63] S. Archambault et al. (PICASSO Collaboration), Constraints on low-mass WIMP interactions on ${ }^{19} F$ from PICASSO, Phys. Lett. B 711, 153 (2012).

[64] E. Behnke et al. (COUPP Collaboration), First dark matter search results from a $4-\mathrm{kg} \mathrm{CF}_{3} \mathrm{I}$ bubble chamber operated in a deep underground site, Phys. Rev. D 86, 052001 (2012). 
[65] M. Felizardo, T. A. Girard, T. Morlat, A. C. Fernandes, A. R. Ramos, J. G. Marques, A. Kling, J. Puibasset et al., Final Analysis and Results of the Phase II SIMPLE Dark Matter Search, Phys. Rev. Lett. 108, 201302 (2012).

[66] M. Ackermann et al. (Fermi-LAT Collaboration), Constraining Dark Matter Models from a Combined Analysis of Milky Way Satellites with the Fermi Large Area Telescope, Phys. Rev. Lett. 107, 241302 (2011).

[67] A. A. Abdo et al. (Fermi-LAT Collaboration), Observations of Milky Way dwarf spheroidal galaxies with the FermiLAT detector and constraints on dark matter models, Astrophys. J. 712, 147 (2010).

[68] J. M. Zheng, Z. H. Yu, J. W. Shao, X. J. Bi, Z. Li, and H. H. Zhang, Constraining the interaction strength between dark matter and visible matter: I. Fermionic dark matter, Nucl. Phys. B854, 350 (2012).

[69] C. Boehm, M. J. Dolan, C. McCabe, M. Spannowsky, and C. J. Wallace, Extended gamma-ray emission from coy dark matter, J. Cosmol. Astropart. Phys. 05 (2014) 009.

[70] T. Sjstrand, S. Ask, J. R. Christiansen, R. Corke, N. Desai, P. Ilten, S. Mrenna, S. Prestel et al., An introduction to PYTHIA 8.2, arXiv:1410.3012.

[71] M. Cacciari, G. P. Salam, and G. Soyez, FastJet user manual, Eur. Phys. J. C 72, 1896 (2012).
[72] V. Khachatryan et al. (CMS Collaboration), Determination of jet energy calibration and transverse momentum resolution in CMS, J. Inst. 6, P11002 (2011).

[73] V. Khachatryan et al. (CMS Collaboration), Performance of the missing transverse energy reconstruction by the CMS experiment in $\sqrt{s}=8 \mathrm{TeV}$ pp data, Reports No. CERNPH-EP-2014-246, No. CMS-PAS-JME-13-003-003.

[74] J. Alwall, M. Herquet, F. Maltoni, O. Mattelaer, and T. Stelzer, MadGraph 5: Going beyond, J. High Energy Phys. 06 (2011) 128.

[75] N. Kidonakis, Next-to-next-to-leading soft-gluon corrections for the top quark cross section and transverse momentum distribution, Phys. Rev. D 82, 114030 (2010).

[76] R. K. Ellis, I. Hinchliffe, M. Soldate, and J. J. van der Bij, Higgs decay to tau + tau-: A possible signature of intermediate mass Higgs bosons at the SSC, Nucl. Phys. B297, 221 (1988).

[77] H. L. Lai, M. Guzzi, J. Huston, Z. Li, P. M. Nadolsky, J. Pumplin, and C.-P. Yuan, New parton distributions for collider physics, Phys. Rev. D 82, 074024 (2010).

[78] R. K. Ellis and G. Zanderighi, Scalar one-loop integrals for QCD, J. High Energy Phys. 02 (2008) 002.

[79] S. D. Badger, E. W. N. Glover, and V. V. Khoze, MHV rules for Higgs plus multiparton amplitudes, J. High Energy Phys. 03 (2005) 023. 